\title{
ROLLING OF COXETER POLYHEDRA ALONG MIRRORS
}

\author{
DMITRI V. ALEKSEEVSKI, PETER W. MICHOR, YURII A. NERETIN
}

\begin{abstract}
The topic of the paper are developments of $n$-dimensional Coxeter polyhedra. We show that the surface of such polyhedron admits a canonical cutting such that each piece can be covered by a Coxeter $(n-1)$-dimensional domain.
\end{abstract}

\section{Introduction. Coxeter groups}

1.1. Coxeter groups in spaces of constant curvature. Consider a Riemannian space $\mathbb{M}^{n}$ of constant curvature, i.e., a Euclidean space $\mathbb{R}^{n}$, a sphere $\mathbb{S}^{n-1}$, or a Lobachevsky space $\mathbb{L}^{n}$ (on geometry of such spaces, see [4).

Let $C \subset \mathbb{M}^{n}$ be an intersection of a finite or locally finite collection of halfspaces 1 .

Consider reflections of $C$ with respect to all $(n-1)$-dimensional faces. Next, consider "new polyhedra" and their reflections with respect to their faces. Etc. The domain $C$ is said to be a Coxeter domain if we get a tiling of the whole space in this way. The group of isometries generated by all such reflections is said to be a reflection group or a Coxeter group (in a narrow sense, see below). We say that a Coxeter group is cocompact if the initial domain $C$ is compact. In this case, we say that $C$ is a Coxeter polyhedron.

Evidently, if $C$ is a Coxeter domain, then the dihedral angles between two neighboring faces of $C$ are of the form $\frac{\pi}{m}$, where $m \geqslant 2$ is an integer. In particular, they are acute, i.e., $\leqslant 90^{\circ}$.

Denote the faces of the polyhedron $C$ by $F_{1}, \ldots, F_{p}$, denote by $s_{1}, \ldots, s_{p}$ the corresponding reflections. Denote by $\pi / m_{i j}$ the angles between adjacent faces. Evidently,

$$
s_{j}^{2}=1, \quad\left(s_{i} s_{j}\right)^{m_{i j}}=1 .
$$

1.2. More terminology. Consider a Coxeter tiling of $\mathbb{M}^{n}$. Below a "chamber" is any ( $n$-dimensional) polyhedron of the tiling. A "face" or "facet" is an $(n-1)$ dimensional face of some chamber; a hyperedge is an $(n-2)$-dimensional edge; a stratum is an arbitrary stratum of codim $\geqslant 1$ of some chamber; a vertex is a vertex.

2000 Mathematics Subject Classification. Primary 51F15, 53C20, 20F55, 22E40.

Key words and phrases. Reflection groups, Coxeter groups, Lobachevsky space, Isometries, Polyhedra, Developments, Trees.

P.W.M. was supported by 'Fonds zur Förderung der wissenschaftlichen Forschung, Projekt P 14195 MAT';

Yu.A.N was supported by Austrian "Fonds zur Förderung der wissenschaftlichen Forschung", project 19064, and also by the Russian Agency on Nuclear Energy, the Dutch fund NWO, grant 047.017.015, and the Japan-Russian grant JSPS-RFBR 07-01-91209.

${ }^{1}$ A natural example with an infinite collection of half-spaces is given on Figure 9 
Also "mirrors" are hyperplanes of reflections. They divide the space $\mathbb{M}^{n}$ into chambers. The group $G$ acts on the set of chambers simply transitively. We denote the reflection with respect to a mirror $Y$ by $s_{Y}$.

Each facet is contained in a unique mirror.

1.3. General Coxeter groups. Take a symmetric $p \times p$ matrix $M$ with positive integer elements, set $m_{j j}=1$; we admit $m_{i j}=\infty$. An abstract Coxeter group is a group with generators $s_{1}, \ldots, s_{n}$ and relations (11).

For such a group we draw a graph (we use the term "Coxeter scheme") in the following way. Vertices of the graph correspond to generators. We connect $i$ and $j$-th vertices by $\left(m_{i j}-2\right)$ edges. In fact, we draw a multiple edge if $k \leqslant 6$, otherwise we write a number $k$ on the edge.

This rule also assign a graph to each Coxeter polyhedron.

1.4. Spherical Coxeter groups. By definition, a spherical Coxeter group, say $\Gamma$, acts by orthogonal transformations of the Euclidean space $\mathbb{R}^{n+1}$. A group $\Gamma$ is said to be reducible if there exists a proper $\Gamma$-invariant subspace in $\mathbb{R}^{n+1}$. Evidently, the orthogonal complement to a $\Gamma$-invariant subspace is $\Gamma$-invariant.

The classification of irreducible Coxeter groups is well known'2, see Bourbaki 8]. The list consists of Weyl groups of semisimple Lie algebras (= Killing's list of root systems) + dihedral groups + groups of symmetries of the icosahedron and 4-dimensional hypericosahedron (the table is given Section 3).

This also gives a classification of reducible groups.

1.5. Coxeter equipments. Next, consider an arbitrary Coxeter polyhedron in $\mathbb{R}^{n}, \mathbb{S}^{n}$, or $\mathbb{L}^{n}$. Consider a stratum $H$ of codimension $k$, it is an intersection of $k$ faces, $H=F_{i_{1}} \cap \cdots \cap F_{i_{k}}$. The reflections with respect to the faces $F_{i_{1}}, \ldots, F_{i_{k}}$ generate a Coxeter group, denote it by $\Gamma(H)=\Gamma\left(F_{i_{1}}, \ldots, F_{i_{k}}\right)$.

This group is a spherical Coxeter group. Namely, for $x \in H$ consider the orthocomplement in the tangent space at $x$ to the stratum $H$ and the sphere in this orthocomplement. Then $\Gamma(H)$ is a reflection group of this Euclidean sphere.

If $H \subset H^{\prime}$, then we have the tautological embedding

$$
\iota_{H^{\prime}, H}: \Gamma\left(H^{\prime}\right) \rightarrow \Gamma(H) .
$$

If $H \subset H^{\prime} \subset H^{\prime \prime}$, then

$$
\iota_{H^{\prime \prime}, H}=\iota_{H^{\prime}, H^{\prime} \iota_{H^{\prime \prime}, H^{\prime}}}
$$

Such a collection of groups and homomorphisms is said to be a Coxeter equipment.

1.6. Cocompact Euclidean Coxeter groups. Here classification is also simple and well known, see Bourbaki [8. Any such group $\Gamma$ contains a normal subgroup $\mathbb{Z}^{n}$ acting by translations and $\Gamma / \mathbb{Z}^{n}$ is a spherical Coxeter group.

\footnotetext{
${ }^{2}$ Actually, these objects were known to Ludwig Schläfli and Wilhelm Killing in XIX century. In 1924, Hermann Weyl identified these groups as reflection groups, in 1934 Harold Coxeter gave a formal classification and also classified Euclidean groups.
} 
1.7. Coxeter groups in Lobachevsky spaces. We report from Vinberg [21, Vinberg, Shvartsman, 22. The situation differs drastically.

a) Coxeter polygons on Lobachevsky plane are arbitrary $k$-gons with angles of the form $\pi / m_{j}$. The sum of exterior angles must satisfy $\sum\left(\pi-\pi / m_{j}\right)>2 \pi$. If $k>5$ this condition holds automatically. For $k=4$ this excludes rectangles, also few triangles are forbidden (in fact, spherical and Euclidean triangles). A Coxeter $k$-gon with prescribed angles depends on $(k-3)$ parameters.

b) In dimensions $n>2$ Coxeter polyhedra are rigid. There are many Coxeter groups in spaces of small dimensions $(n=3,4,5)$, but for $n \geqslant 30$ there is no Coxeter group with compact fundamental polyhedron at all. For $n>996$ there is no Coxeter group of finite covolume (Prokhorov, Khovanskii, 1986, see [13]); the maximal dimensions of known examples are: 8 for compact polyhedra (Bugaenko), and 21 for a polyhedron of finite volume (Borcherds). For $n=3$ there is a nice Andreev's description [5] of all Coxeter polyhedra, it is given in the following two subsections.

1.8. Acute angle polyhedra in $\mathbb{L}^{3}$. First, we recall the famous (and highly nontrivial) Steinitz Theorem (see, e.g., [15]) about possible combinatorial structure of convex polyhedra in $\mathbb{R}^{3}$.

Since the boundary of a polyhedron is a topological sphere $S^{2}$, edges form a connected graph on the sphere, it divides the sphere into polygonal domain (we use the term 'face' for such a domain). There are the following evident properties of the graph:

- each edge is contained in 2 faces;

- each face has $\geqslant 3$ vertices;

- the intersection of any pair of faces can by the empty set, a vertex, or an edge.

Theorem. (Ernst Steinitz) Any graph on the sphere $S^{2}$ satisfying the above conditions can be realized as a graph of edges of a convex polyhedron.

Our next question is the existence of a convex polyhedron in $\mathbb{L}^{3}$ of a given combinatorial structure where each dihedral (i.e., between two adjacent faces) angle is a given acute angle ('acute' or also 'non-obtuse' means $\leqslant \pi / 2$ ) There are the following a priori properties of such polyhedra:

1) All spatial angles are simplicial, i.e., each vertex of the graph is contained in 3 edges. The angles $\varphi_{1}, \varphi_{2}, \varphi_{3}$ in a given vertex satisfy

$$
\varphi_{1}+\varphi_{2}+\varphi_{3}>2 \pi \text {. }
$$

2) At each vertex, the set of all dihedral angles determines all other angles in each face at this vertex (by the spherical cosine theorem). A face must be a Lobachevsky polygon, i.e., the sum of its exterior angles must be $\geqslant 2 \pi$. Since all dihedral angles are acute, angles in each face are also acute. Therefore our conditions forbid only rectangles and some triangles.

3) The following restriction is non-obvious: We say that a $k$-prismatic element of a convex polyhedron $C$ is a sequence

$$
F_{1}, \quad F_{2}, \quad \ldots, \quad F_{k}, \quad F_{k+1}:=F_{1}
$$




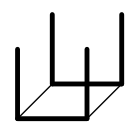

Figure 1. The following configuration with dihedral angles $=\pi / 2$ on thick edges is forbidden in the Andreev Theorem. In this case, we would get a quadrangle with right angles, but such quadrangles do not exist in Lobachevsky space.

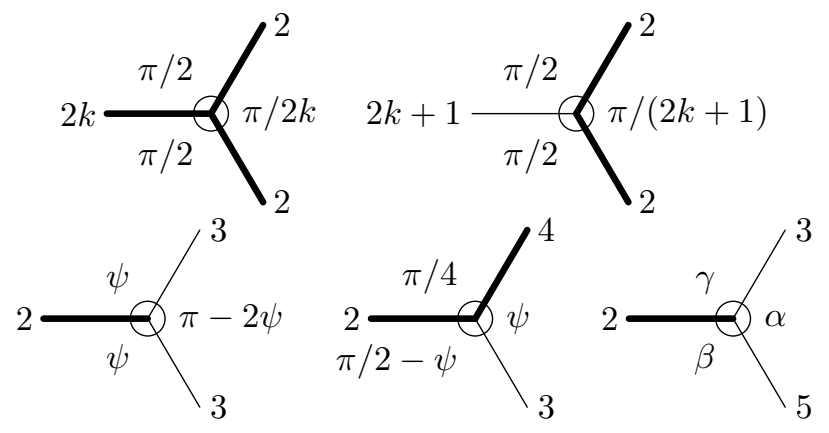

Figure 2. We draw all possible types of vertices of an Andreev polyhedron. We present the labels $m_{j}$ on the edges and flat angles in faces. Here $\psi=\arctan \sqrt{2}$ and $\alpha, \beta, \gamma$ are explicit angles with $\alpha+\beta+\gamma=\pi / 2$. Evaluations of all these angles are given in figures in Section 2 ,

We draw a thick line iff the label is even.

of faces such that $F_{k}$ and $F_{k+1}$ have a common edge, and all triple intersections $F_{i} \cap F_{j} \cap F_{k}$ are empty.

Lemma. (Andreev) For any prismatic element in an acute angle polyhedron, the sum of exterior dihedral angles is $>2 \pi$.

Theorem. (Andreev) Consider a Steinitz-admissible 3-valent spherical graph with $>4$ vertice 3 . Prescribe a dihedral acute angle to each edge in such a way that:

- the inequality (2) in each vertex is satisfied;

- all 3- and 4-prismatic elements satisfy the previous lemma;

- we forbid the configuration given on Figure 1 .

Under these assumptions, there exists a unique convex polyhedron $\subset \mathbb{L}^{3}$ of the given combinatorial structure and with the given acute angles.

The uniqueness is a rigidity theorem of Cauchy type (see [1, [15]). The existence is a deep unusual fact; it is a special case of a theorem of Aleksandrov type [1] obtained by Rivin, see [16], [14.

For some applications of the Andreev and Rivin Theorems to elementary geometry, see Thurston [19], Rivin [17].

\footnotetext{
${ }^{3}$ Simplices are exceptions. However, their examination is simple, Lanner, 1950, see, e.g., 22 .
} 
1.9. Andreev polyhedra. Andreev's Theorem provides us a description of all Coxeter polyhedra in $\mathbb{L}^{3}$. Now all angles have the form $\pi / m_{i j}$ with integer $m_{i j}>1$. We simply write the labels $m_{i j}$ on the corresponding edges.

Below the term "Andreev polyhedron" will mean a compact Coxeter polyhedron in $\mathbb{L}^{3}$.

All possible pictures at vertices of Andreev polyhedra are given in Figure 2 .

1.10. Results of the paper. Consider a convex polyhedron $C$ in a space $\mathbb{M}^{n}$ of constant curvature. Following Alexandrov [1, we regard the boundary $\Xi=\partial C$ of $C$ as an $(n-1)$-dimensional manifold of constant curvature with singularities. In the case $n=3$, we get a two-dimensional surface with conic singularities of negative curvature (see e.g. Figure 2 in all the cases the sum of angles at a singularity is $<2 \pi)$.

Now, cut $\Xi$ along hyperedges with even labels (i.e., hyperedges with dihedral angles $\pi / 2 k)$. Let $\Omega_{1}, \Omega_{2}, \ldots$ be the connected pieces of the cut surface.

Theorem 1.1. The universal covering $\Omega_{j}^{\sim}$ of each $\Omega_{j}$ is a Coxeter domain in $\mathbb{M}^{n-1}$.

Proof for Andreev polyhedra. We simply look to Figure 2, In all the cases, angles between thick edges are Coxeter.

We also describe tilings of mirrors, groups of transformations of mirrors induced by the initial Coxeter group (Theorem 2.10) and the Coxeter equipments of $\Omega_{j}^{\sim}$ (Theorem 3.2).

The addendum to the paper contains two examples of 'calculation' of developments, for an Andreev prism $\subset \mathbb{L}^{3}$ and for a Coxeter simplex $\subset \mathbb{L}^{4}$. The proof of the Andreev Theorem is nonconstructive. In various explicit cases, our argumentation allows to construct an Andreev polyhedron from the a priori information about its development. Our example illustrates this phenomenon.

On the other hand, there arises a natural problem of elementary geometry:

- Which Andreev polyhedra are partial developments of 4-dimensional Coxeter polyhedra? Is it possible to describe all 3-dimensional polyhedra that are faces of 4-dimensional Coxeter polyhedra?

Our main argument (Rolling Lemma 2.1) is very simple, it is valid in a wider generality, we briefly discuss such possibilities in the next two subsections.

\subsection{Polyhedral complexes and projective Coxeter polyhedra.}

Theorem. (Tits) Any Coxeter group can be realized as a group of transformations of an open convex subset of a real projective space $\mathbb{R P}^{n}$ which is generated by a collection of reflections $s_{1}, \ldots, s_{p}$ with respect to hyperplane 4 intersecting the subset. The closure of a chamber is a convex polyhedron.

See also Vinberg [20].

\footnotetext{
4 a reflection is determined by a fixed hyperplane and a reflected tranversal line
} 

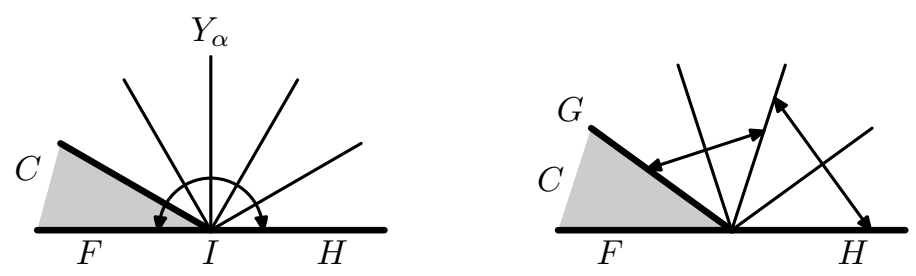

Figure 3. Even and odd labels. Proof of Rolling Lemma.

1.12. A more general view. Nikolas Bourbak 5 proposed a way to build topological spaces from Coxeter groups. M. Davis used this approach in numerous papers (see e.g. 99, 10]) and the book [11; in particular he constructed nice examples/counterexamples in topology.

Also it is possible to consider arbitrary Riemannian manifolds equipped with a discrete isometric action of a Coxeter group such that the set of fixed points of each generator is a (totally geodesic) hypersurface, and such that the generators act as reflections with respect to these submanifolds. In this context, a chamber itself can be a topologically non-trivial object, see [11, [3].

\section{Rolling OF CHAMBer}

In this section, $\mathbb{M}^{n}$ is a space $\mathbb{L}^{n}, \mathbb{S}^{n}, \mathbb{R}^{n}$ of constant curvature equipped with a Coxeter group $\Gamma$ or, more generally, any space described in Subsection 1.11.

Fix a mirror $\mathbb{X}^{n-1}$ in $\mathbb{M}^{n}$. Consider intersections of $\mathbb{X}^{n-1}$ with other mirrors $Y_{\alpha}$. The set $\mathbb{X}^{n-1} \backslash \bigcup Y_{\alpha}$ is a disjoint union of open facets. Thus, we get a tiling of $\mathbb{X}^{n-1}$ by facets.

Our aim is to describe this tiling in the terms of the geometry of a chamber.

\subsection{Rolling lemma.}

Lemma 2.1. Let $I \subset \mathbb{X}^{n-1}$ be an $(n-2)$-dimensional hyper-edge of our tiling. Let $F, H \subset \mathbb{X}^{n-1}$ be the facets adjacent to $I$.

a) If the label $m_{I}$ of $I$ is even, then $I$ is contained in a certain mirror $Y_{\alpha}$ orthogonal to $X$. In particular $s_{Y_{\alpha}} F=H$.

b) Let the label $m$ be odd. Let $C$ be a chamber adjacent to the facet $F$. Let $G$ be another facet of $C$ adjacent to the same hyper-edge $I$. Then $G$ is isometric to $H$. More precisely, there is $\gamma \in \Gamma$ fixing all the points of $I$ such that $\gamma G=H$.

Proof is given in figure 3 ,

2.2. Algorithm generating the tiling. Let $C$ be a chamber adjacent to a facet $F \subset \mathbb{X}^{n-1}$. Consider an hyper-edge $I$ of $C$ lying in $\mathbb{X}^{n-1}$.

Operation 1. Let the hyper-edge $I$ be odd. Consider a facet $G \neq F$ of $C$ adjacent to $I$, consider the corresponding $\gamma$ from Lemma 2.1 and draw $\gamma G$ on $\mathbb{X}^{n-1}$

Operation 2. If the hyper-edge $I$ is even, then we reflect $F$ in $\mathbb{X}^{n-1}$ with respect to $I$.

We perform all the possible finite sequences of such operations. By the Rolling Lemma, we get the whole tiling of the mirror $\mathbb{X}^{n-1}$.

\footnotetext{
${ }^{5}$ Apparently, he used the work by Jacque Tits [18; the latter text is inaccessible for the authors.
} 

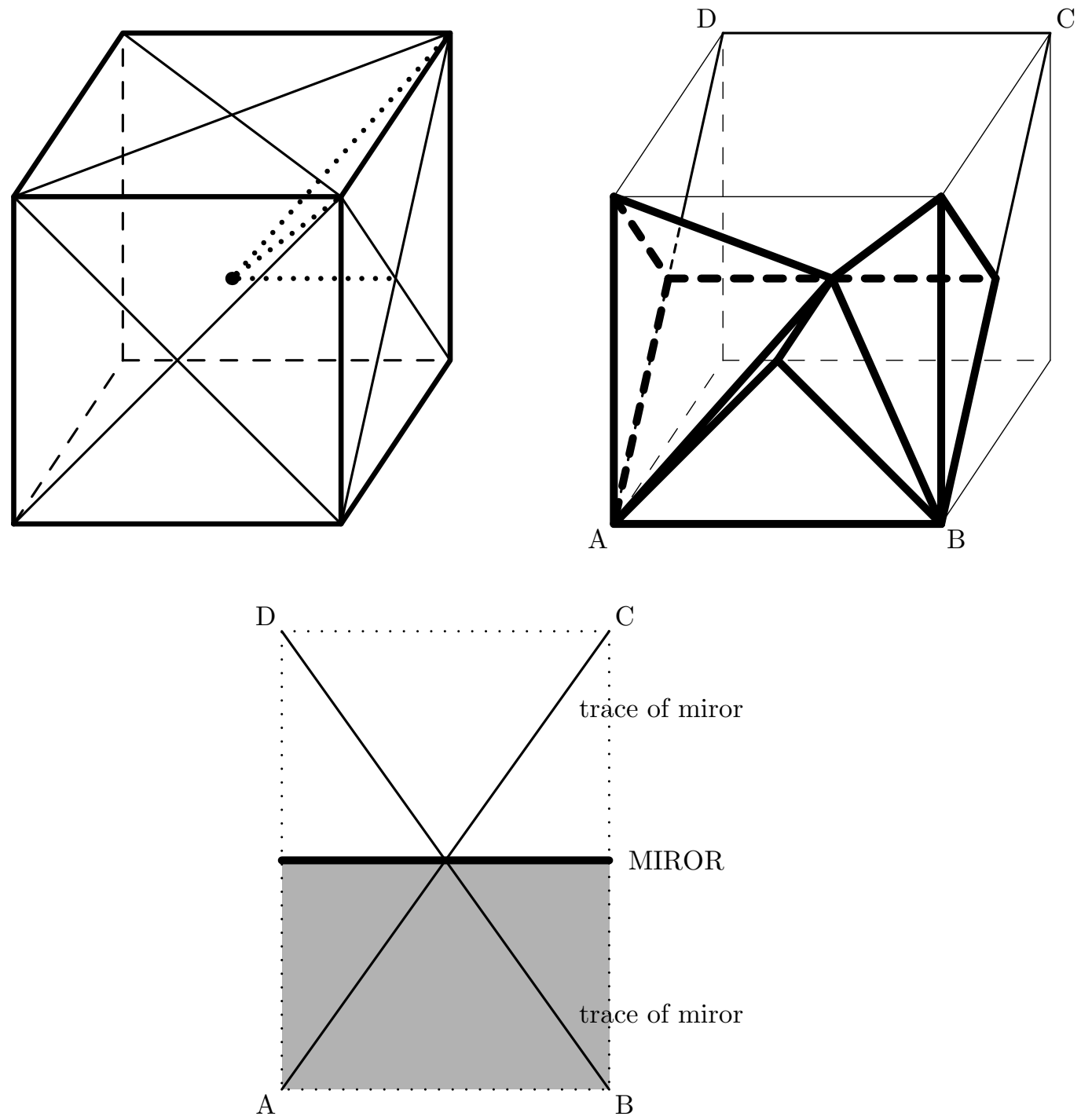

Figure 4. Example of rolling: the reflection group $A_{3}$ in $\mathbb{R}^{3}$. The mirrors are planes passing through opposite edges of the cube. There are 24 Weyl chambers, which are simplicial cones with dihedral angles $\pi / 3, \pi / 3, \pi / 2$ (we draw them as simplices). Rolling of a Weyl chamber by the mirror $A B C D$ produces a half-plane.

We can also regard $A_{3}$ as a reflection group on the 2-dimensional sphere $\mathbb{S}^{2}$.

REMARK. Let $\mathbb{M}^{n}=\mathbb{R}^{3}, \mathbb{S}^{3}, \mathbb{L}^{3}$ be a usual 3-dimensional space of constant curvature. Operation 1 corresponds to rolling of a polyhedron $C$ along the hyperplane $\mathbb{X}^{n-1} \sim \mathbb{M}^{2}$ over the edge $I$. 


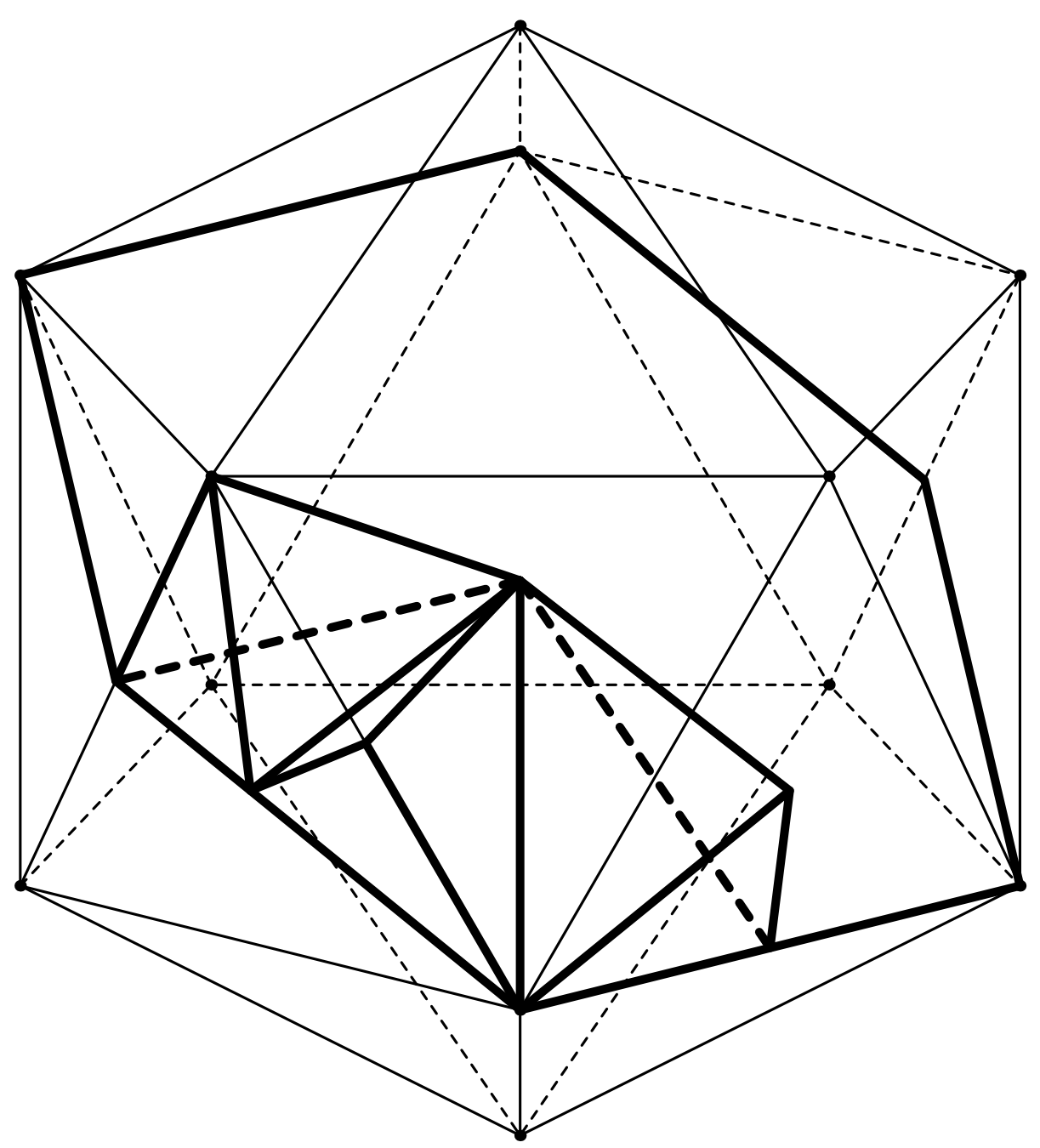

Figure 5. Example of rolling: the icosahedral group $\mathrm{H}_{3}$. It is generated by reflections with respect to bisectors of segments connecting midpoints of opposite edges of the icosahedron. The bisectors separate $\mathbb{R}^{3}$ into 120 simplicial cones with dihedral angles $\pi / 2, \pi / 3, \pi / 5$. In the figure the simplicial cones are cut by the surface of the icosahedron.

We show an admissible rolling of a Weyl chamber along a mirror. The final chamber in the mirror is a quadrant.

2.3. The group preserving the mirror $\mathbb{X}^{n-1}$. For a mirror $\mathbb{X}^{n-1}$, consider the group $\Gamma_{*}=\Gamma_{*}\left(\mathbb{X}^{n-1}\right)$ of all the isometries of $\mathbb{X}^{n-1}$ induced by elements of $\Gamma$ preserving $\mathbb{X}^{n-1}$.

If $\gamma \in \Gamma$ preserves $\mathbb{X}^{n-1}$, then $s_{\mathbb{X}^{n-1}} \gamma$ also preserves $\mathbb{X}^{n-1}$ and agrees with $\gamma$ on $\mathbb{X}^{n-1}$. Thus each element of $\Gamma_{*}$ is induced by two different elements of $\Gamma$. 

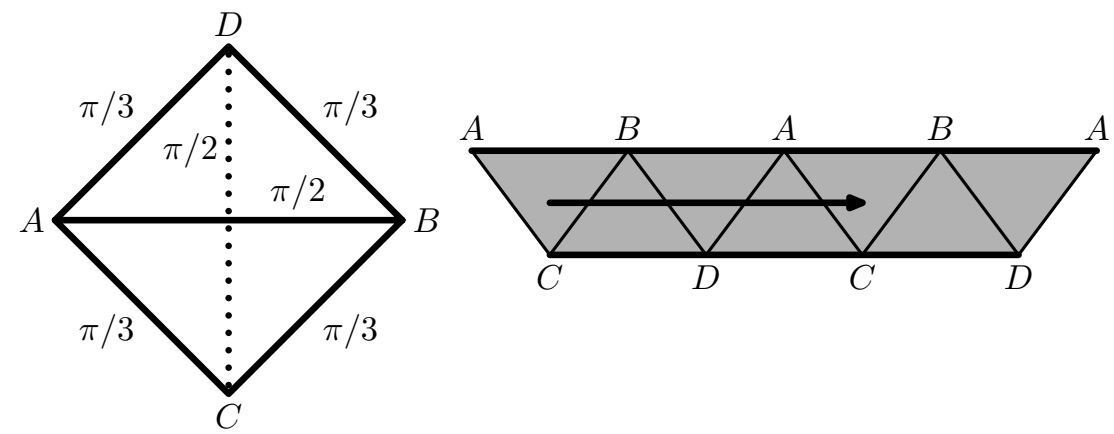

FIgURE 6. Example of rolling: the (affine) Euclidean reflection group $\widetilde{A}_{4}$ in $\mathbb{R}^{3}$. A chamber is the simplex $A B C D$. Rolling through $A B$ and $C D$ is forbidden. Deleting these edges from the surface of the simplex, we get a non-simply connected surface. Hence, the process of rolling is infinite. The arrow shows the deck transformation induced by the generator of the fundamental group.
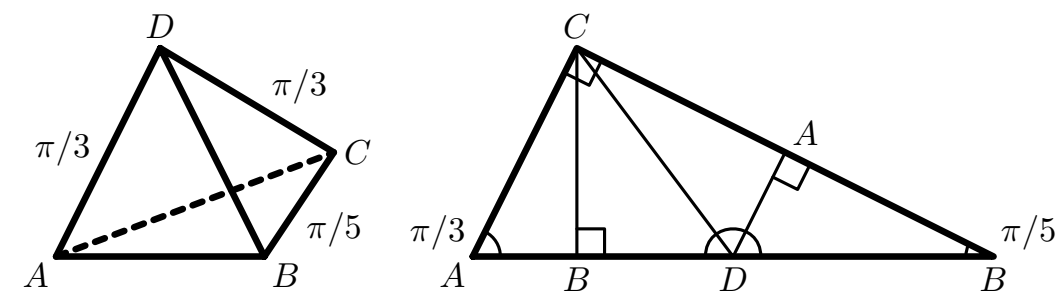

FiguRE 7. Example of rolling: the hypericosahedral group $\mathrm{H}_{4}$ acting on the 3 -dimensional sphere $\mathbb{S}^{3}$. The chamber is is the spherical simplex drawn in the figure (we omit all labels $\pi / 2$ on edges).

The angle $=\pi$ on the development at $D$ was evaluated in figure 4. The right angle at $C$ was evaluated in figure [5.

The spherical triangle $A B C$ is present on the circumscribed sphere in the next figure (in spite of the absence of the sphere itself).

Observation 2.2. Let $F_{1}, F_{2} \subset \mathbb{X}^{n-1}$ be equivalent facets. There is a unique element $\mu \in \Gamma_{*}\left(\mathbb{X}^{n-1}\right)$ such that $\mu F_{1}=F_{2}$.

2.4. Reflections in mirrors and the new chamber. Consider all the mirrors $Z_{\alpha} \subset \mathbb{M}^{n}$ orthogonal to our mirror $\mathbb{X}^{n-1}$. The corresponding reflections $s_{Z_{\alpha}}$ generate a reflection group on $\mathbb{X}^{n-1}$; denote this group by $\Delta=\Delta\left(\mathbb{X}^{n-1}\right)$.

Observation 2.3. $\Delta$ is a normal subgroup in $\Gamma_{*}\left(\mathbb{X}^{n-1}\right)$.

Indeed, if $s$ is a reflection, then $\gamma^{-1} s \gamma$ is a reflection.

Consider a chamber $C$ of $\mathbb{M}^{n}$ lying on $\mathbb{X}^{n-1}$ (i.e., having a facet in $\mathbb{X}^{n-1}$ ) and consider all possible sequences of admissible rollings, i.e., we allow Operation 1 of Algorithm 2.2 and we forbid Operation 2. Denote by $B \subset \mathbb{X}^{n-1}$ the domain 


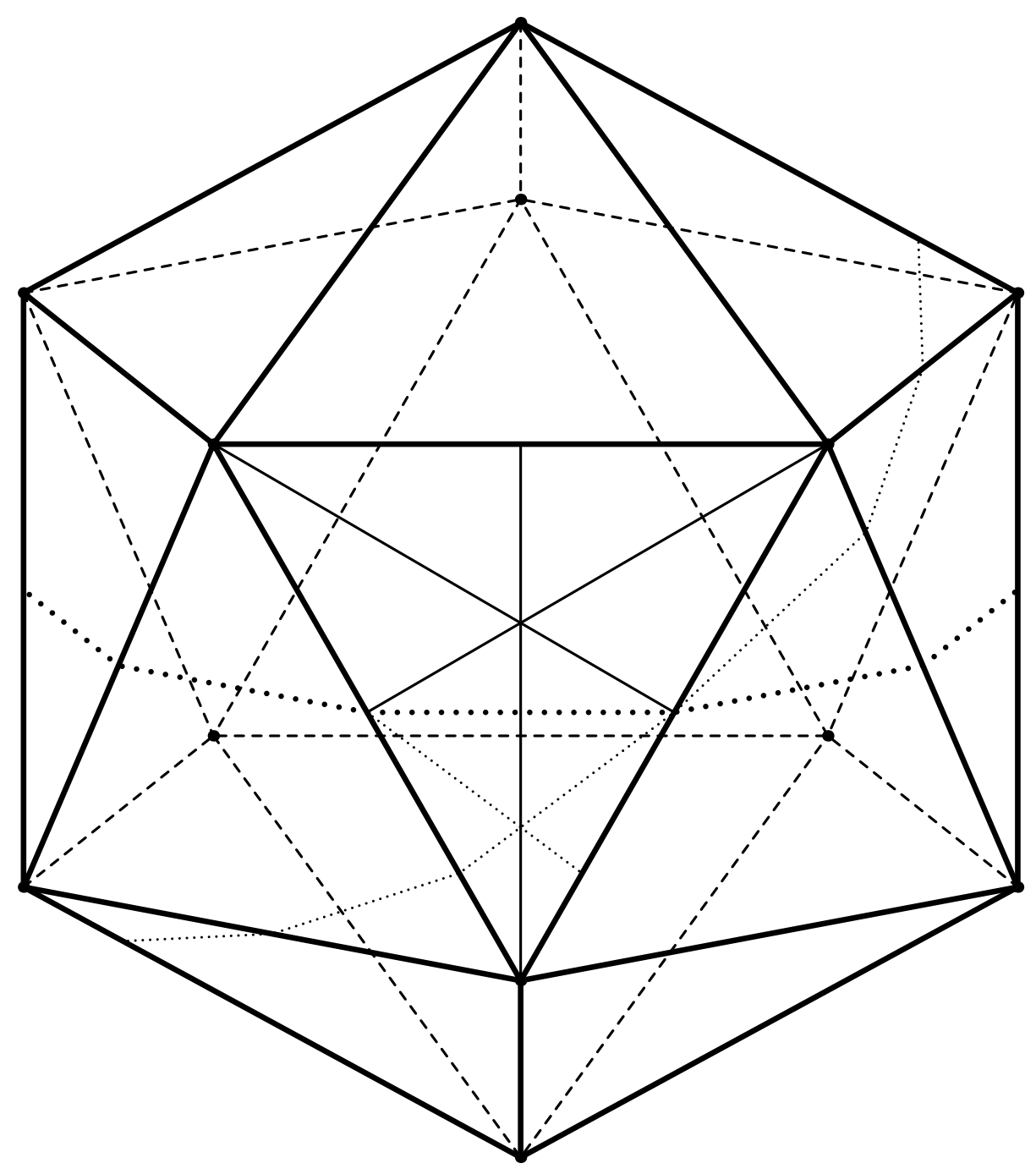

FiguRE 8. Example of rolling: the hypericosahedral group $\mathrm{H}_{4}$ acting in $\mathbb{R}^{4}$. The figure presents the tiling of a mirror, i.e., of $\mathbb{R}^{3}$, by simplicial cones. We draw intersections of simplicial cones with the boundary of the icosahedron. Consider 3 types of 'axes' of the icosahedron:

A) segments connecting midpoints of opposite edges;

B) segments connecting central points of opposite faces;

C) diagonals connecting opposite vertices.

Consider bisectors of all such segments. Type A bisectors are mirrors. They divide $\mathbb{R}^{3}$ into 120 simplicial chambers. Six chambers are presented in the front face of the icosahedron.

Adding bisectors of type $\mathrm{B}$ and $\mathrm{C}$ we obtain a partition of $\mathbb{R}^{3}$ into 480 simplicial cones. This is the desired tiling.

In this figure, we present subdivisions of two chambers. A proof of this picture is contained on figure 7 

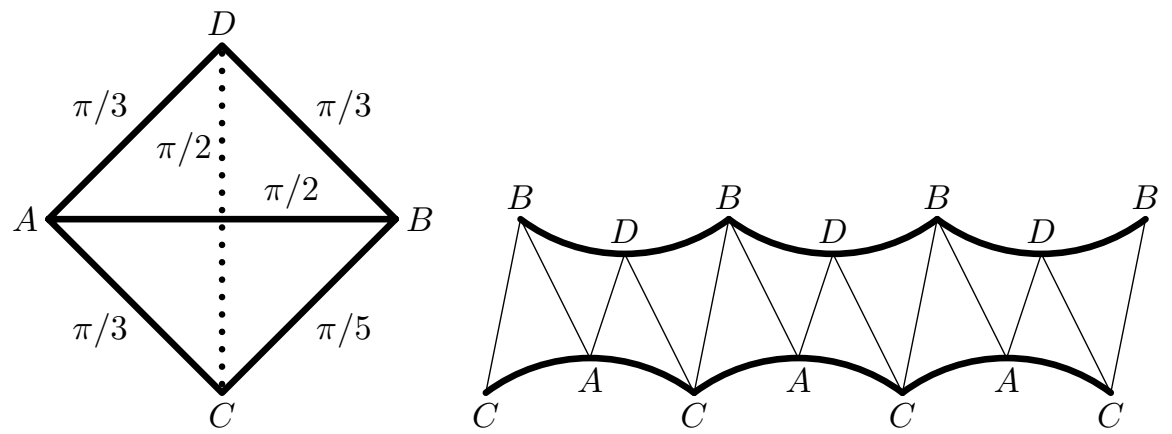

Figure 9. Example. A Coxeter simplex in $\mathbb{L}^{3}$. Its development is an infinite 'strip' $\subset \mathbb{L}^{2}$ bounded by two infinite polygonal curves, interior angles between segments of polygonal curves are $\pi / 2$ and $\pi$.

obtained by rolling, tiled by the traces of facets of $C$ making contact with $\mathbb{X}^{n-1}$ during rolling.

Theorem 2.4. $B$ is a chamber of the reflection group $\Delta\left(\mathbb{X}^{n-1}\right)$.

Proof. We can not roll further if and only if we meet a "vertical" mirror.

EXAMPLES OF ROLLING. Some examples of rolling corresponding to the usual spherical Coxeter groups

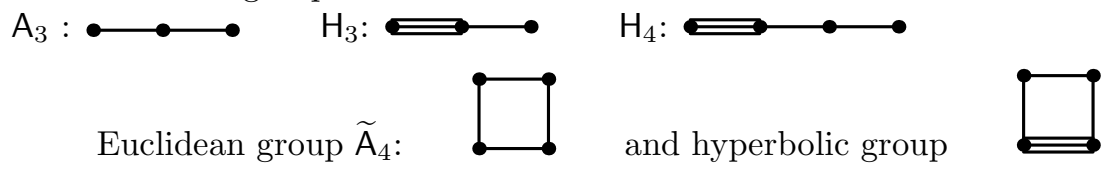

are given in figure 449. In these figures, we also evaluate the new chamber $B$.

Lemma 2.5. Each $(n-3)$-dimensional stratum of our tiling of $\mathbb{X}^{n-1}$ is contained in a mirror of the group $\Delta\left(\mathbb{X}^{n-1}\right)$.

Proof. This stratum is equipped with a finite 3-dimensional Coxeter group (i.e., $A_{3}, B_{3}, H_{3}, A_{1} \oplus G_{2}^{m}, A_{1} \oplus A_{1} \oplus A_{1}$, see Table below). For each mirror of such a group there exists an orthogonal mirror.

2.5. Rolling scheme. Denote by $\Xi(C)$ the surface of the initial chamber $C$, let $\Xi^{\prime}(C)$ be the surface with all even edges deleted.

Lemma 2.6. $\Xi^{\prime}$ does not contain $(n-3)$-dimensional strata of $C$.

This is rephrasing of Lemma 2.5.

Consider the graph, whose vertices are the facets of $\Xi^{\prime}$; vertices are connected by an edge if the corresponding facets are neighbors in $\Xi^{\prime}$. We call this graph the Rolling scheme. In fact, the Rolling scheme is the Coxeter scheme 1.3 with removed even (and infinite) edges.

Proposition 2.7. The surface $\Xi^{\prime}$ is homotopically equivalent to the Rolling scheme. 
a)

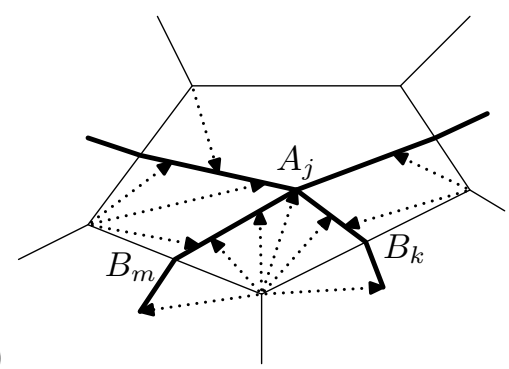

b)

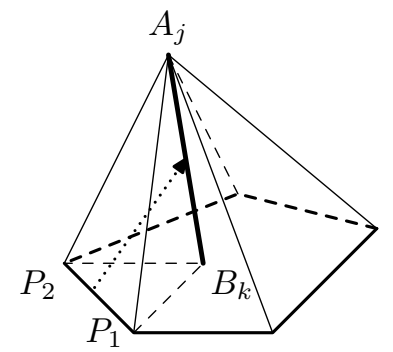

Figure 10. Proof of Lemma 2.8.

a) $n=3$. Graph on a surface of a 3 -dimensional polytop and a retraction. Recall that we have removed vertices.

b) $n=4$. A piece of a 3 -face of 4-dimensional polyhedron. Recall that 1-dimensional edges are removed. Inside a simplex $P_{1} P_{2} A_{j} B_{k}$ the retraction is the projection to $A_{j} B_{k}$ with center on the segment $P_{1} P_{2}$. Note that all segments connecting $A_{j} B_{k}$ and $P_{1} P_{2}$ are pairwise non-intersecting.

2.6. Proof of Proposition 2.7, Let $U$ be a convex polyhedron in $\mathbb{R}^{n}$, denote by $\Xi$ its surface. Choose a point $A_{j}$ in interior of each $(n-1)$-dimensional face. Choose a point $B_{k}$ in interior of each $(l-2)$-dimensional boundary stratum (hyperedge) of $U$.

Draw the segment $\left[A_{j}, B_{k}\right]$ iff the face contains the hyperedge. Thus we get a graph $T$ on the surface of the polyhedron $C$ whose vertices are enumerated by faces of $U$ and edges are enumerated by hyperedges of $U$. Denote by $\Xi \nabla$ the surface of the polyhedron $S$ without boundary strata of dimension $(n-3)$.

Lemma 2.8. The graph $T$ is a deformation retract of $\Xi \nabla$. Moreover, it is possible to choose a homotopy that preserves all faces and all hyperedges.

Proof. See figure 10.

Proposition 2.7 follows from Lemma 2.8 .

2.7. Action of the fundamental group on mirror. Let $F$ be a facet in $\mathbb{X}^{n-1}$, let $C$ be a chamber of $\mathbb{M}^{n}$ lying on $F$, and let $B \supset F$ be the chamber of the reflection group $\Delta\left(\mathbb{X}^{n-1}\right)$ obtained by rolling $C$, as described in subsection 2.4 .

Let $\Omega$ be a connected component of $\Xi^{\prime}$ containing the facet $F$.

Let $F_{1}, \ldots, F_{r}$ be facets $\subset \Omega$. We can think that each facet has its own color; thus the mirror $\mathbb{X}^{n-1}$ is painted in $r$ colors. Moreover, for each facet $H \in \mathbb{X}^{n-1}$ there is a canonical bijection ('parametrization') from the corresponding $F_{i} \subset \Omega$ to $H$. We say that a bijection $\mathbb{X}^{n-1} \rightarrow \mathbb{X}^{n-1}$ (or $B \rightarrow B$ ) is an isomorphism if it preserves the coloring and commutes with the parameterizations.

Proposition 2.9. a) The chamber $B \subset \mathbb{X}^{n-1}$ is the universal covering of $\Omega$.

b) Any deck transformations of $B$ is an isomorphism $B \rightarrow B$ and admits a unique extension to an isomorphism of the mirror $\mathbb{X}^{n-1}$.

c) Each isomorphism $\mu \in \Gamma_{*}\left(\mathbb{X}^{n-1}\right)$ preserving $B$ is induced by a deck transformation. 


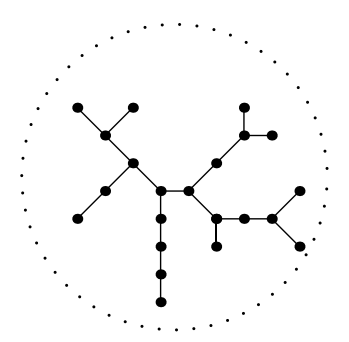

Figure 11. A graph of vicinity of $(n-1)$-dimensional facets in the new $(n-1)$-dimensional chamber $B$ is a tree.

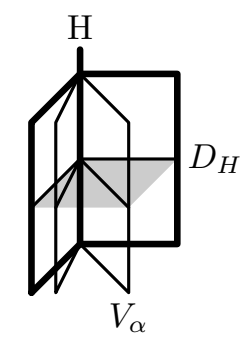

FIgURE 12. Subdivision of the cone normal to a stratum.

Proof. a) Denote by $\Omega^{\sim}$ the universal covering of $\Omega$. The chamber $B$ was constructed as the image of $\Omega^{\sim}$. Moreover, the map $\Omega^{\sim} \rightarrow C$ is locally bijective. On the other hand, a chamber on a simply connected manifold is simply connected see (see [3, 2.14); therefore $B \simeq \Omega^{\sim}$.

b) A deck transformation $B \rightarrow B$ is an isometry by the rolling rules. Let a deck transformation send a facet $F$ to $F^{\prime}$. Then the facets $F, F^{\prime}$ are $\Gamma$-equivalent, and the corresponding map in $\Gamma$ is an isometry of $\mathbb{X}^{n-1}$.

c) Let $F \subset \mathbb{X}^{n-1}$ be a facet. We take the deck transformation sending $F$ to $F^{\prime}$.

\subsection{Description of $\Gamma_{*}\left(\mathbb{X}^{n-1}\right)$.}

Theorem 2.10. The group $\Gamma_{*}\left(\mathbb{X}^{n-1}\right)$ is a semidirect product $\operatorname{Deck}(B) \ltimes \Delta\left(\mathbb{X}^{n-1}\right)$.

Proof. Indeed, the group $\Delta\left(\mathbb{X}^{n-1}\right)$ acts simply transitively on the set of chambers in $\mathbb{X}^{n-1}$; the group $\operatorname{Deck}(B)$ acts simply transitively on the set of facets of a given type in the chamber $B$.

\section{Reduction of EQUiPMENT}

We keep the notation of the previous section. Our aim is to describe the Coxeter equipment of the new chamber $B$.

3.1. Combinatorial structure of the tiling of the chamber. Consider a graph $\mathfrak{F}$ whose vertices are enumerated by $(n-1)$-facets lying in $B$, two vertices are connected by an edge if they have a common $(n-2)$-dimensional stratum (a former hyperedge in $\mathbb{M}^{n}$ ).

Observation 3.1. $\mathfrak{F}$ is a tree. 
Table. Reduction of spherical Coxeter schemes

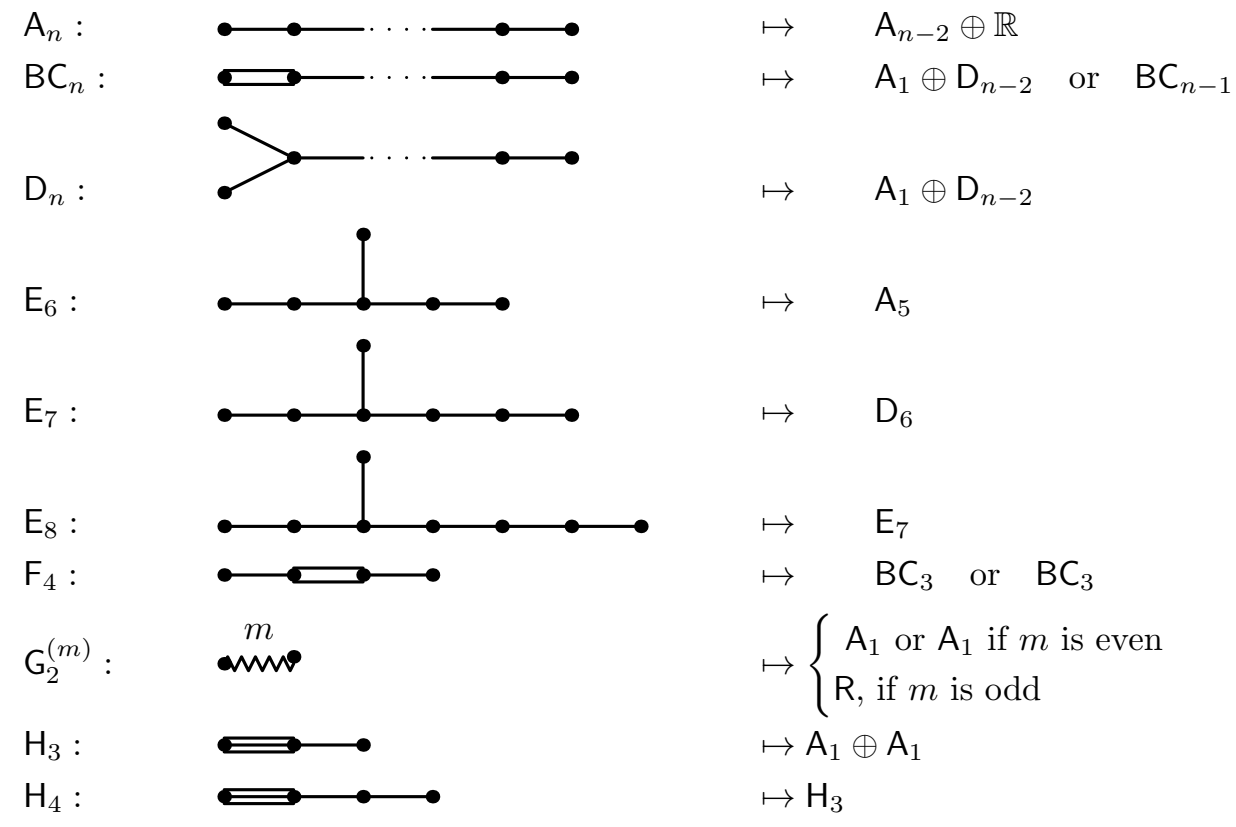

Proof. Indeed, the universal covering of a graph is a tree.

If the initial rolling scheme is a tree, then we get the same tree. If the rolling scheme contains a cycle, then we get an infinite tree (examples: Figures 6, 9, the rolling schemes contain 1 cycle).

3.2. New equipment. All the strata of $B$ of dimension $<(n-2)$ are contained in the boundary of $B$. These strata of $B$ have their own equipments (in the sense of the Coxeter manifold $\mathbb{X}^{n-1}$ ).

For a boundary stratum $H$ of $B$ and some point $y \in H$, denote by $N_{H} \subset T_{y} \mathbb{X}^{n-1}$ the normal subspace to $H \subset \mathbb{X}^{n-1}$. The normal cone $D_{H} \subset N_{H}$ is the cone consisting of vectors looking inside $B$. Some of $(n-2)$-dimensional strata (former hyperedges) $V_{\alpha}$ contain $H$ and thus we get the subdivision of the normal cone $D_{H}$ by tangent spaces to $(n-2)$-dimensional strata, see figure 12 .

We wish to describe the equipment of $B \subset \mathbb{X}^{n-1}$ and the subdivisions of normal cones $D_{H}$.

3.3. Finite Coxeter groups. Let $\Gamma$ be a finite Coxeter group acting in $\mathbb{R}^{n}$. Let $\mathbb{X}_{j}^{n-1}$ be the mirrors, let $v_{j}$ be the vectors orthogonal to the corresponding mirrors. For a vector $v_{k}$ denote by $R=R_{k}$ the set of all $i$ such that $v_{i}$ is orthogonal to $v_{k}$.

The reflection group $\Delta\left(\mathbb{X}_{k}^{n-1}\right)$ is generated by reflections with respect to mirrors $\mathbb{X}_{i}^{n-1}$, where $i$ ranges in $R$.

A. Let the Coxeter group $\Gamma$ be irreducible. We come to the list given in the Table. Some comments: 
1) $\mathrm{G}_{2}^{(m)}$ denotes the group of symmetries of a regular plane $m$-gon, $\mathrm{R}$ denotes the one-element group acting in $\mathbb{R}^{1}$; all other notations are standard, see $[\underline{8}$.

2) In some cases, there are two $\Gamma$-nonequivalent mirrors, then we write both possible variants.

The rolling scheme (see 2.5) is the Coxeter scheme without even edges.

ExAmPle. a) For the Weyl chamber $\mathrm{E}_{8}$, its complete development is the Weyl chamber $\mathrm{E}_{7}$.

b) For the Weyl chamber $\mathrm{BC}_{n}$, one of the facets is the Weyl chamber $\mathrm{BC}_{n-1}$. All the remaining facets are connected by the rolling graph; the development is the Weyl chamber $\mathrm{A}_{1} \oplus \mathrm{D}_{n-2}$.

ProOF OF TABLE is a case-by-case examination of root systems; for the groups $\mathrm{H}_{3}$ and $\mathrm{H}_{4}$ the proofs are given Figures 5, 7, , 8 (on the other hand the reader can find a nice coordinate description of the hypericosahedron in 8 .

B. If the Coxeter group $\Gamma$ be reducible,

$$
\Gamma=\Gamma_{1} \times \Gamma_{2} \times \ldots
$$

then its Weyl chamber is the product of the Weyl chambers for the corresponding chambers $C=C_{1} \times C_{2} \times \ldots$ The Coxeter scheme of $\Gamma$ is the union of the Coxeter schemes of $\Gamma_{j}$, hence the rolling graph of $\Gamma$ is the union of the rolling graphs for all the $\Gamma_{j}$. Now we reduce one of factors $C_{j} \mapsto B_{j}$ according to the rules given in the Table, and we get a Weyl chamber $B_{j} \times \prod_{i \neq j} C_{i}$.

3.4. Reduction of equipment. Let $H$ be an $(n-k)$-dimensional stratum of $C$ $(k \geqslant 3)$, let $\Gamma_{H}(C)$ be the corresponding Coxeter group, and let $\mathfrak{N}_{H}(C)$ be its chamber in the normal cone. Denote by $\Gamma_{H}(B)$ the corresponding group of the equipment of $B$ and by $\mathfrak{N}_{H}(B)$ the corresponding chamber in the normal cone.

Theorem 3.2. The group $\Gamma_{H}(B)$ is obtained by reduction of the group $\Gamma_{H}(B)$ and the subdivision of $\mathfrak{N}_{H}(B)$ is a partial development of the Weyl chamber $\mathfrak{N}_{H}(C)$

Proof is obvious. We consider rollings of $C$ with fixed hyperedge $H$. The subdivision of the cone $D_{H}$ is obtained by rolling with respect to the hyperedges containing $H$.

\section{Addendum. Elementary geometry of Andreev polyhedra}

4.1. Rolling of Andreev polyhedra and billiard trajectories in Coxeter polygons. Firstly, our construction gives some information about developments of Andreev polyhedra.

Let us roll an Andreev polyhedron $\subset \mathbb{L}^{3}$ along a mirror $\simeq \mathbb{L}^{2}$. In this case, the chamber $B$ of a mirror is a convex plane Coxeter domain. By construction, $B$ is subdivided into several convex polygons by a certain family of lines.

Proposition 4.1. All the possible variants of meetings of lines of the subdivision and the boundary of $B$ are presented in figure 13 .

Proof. We watch all the possible variants of reduction of 3-dimensional finite Coxeter groups to a mirror. The parts a), b), c), d) of figure 13 correspond to $\mathrm{G}_{2}^{2 k+1}, \mathrm{BC}_{3}, \mathrm{~A}_{3}=\mathrm{D}_{3}, \mathrm{H}_{3}$, respectively. 


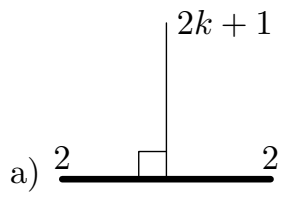

b)

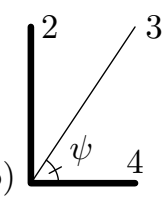

c)
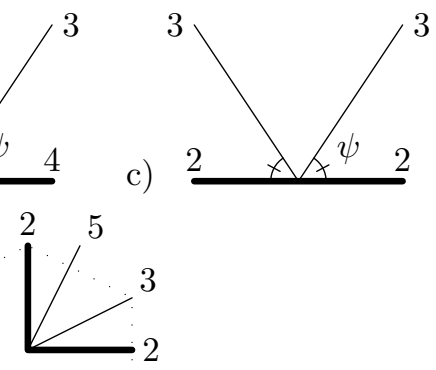

d)

Figure 13. Subdivisions of a Coxeter polygon on the Lobachevsky plane (we also draw the labels on lines). There only 4 possible variants of meetings between lines of a subdivision and the boundary. Here $\tan \psi=\sqrt{2}$; for case d) see figure 5 .

In cases c) and d), the corresponding trihedral angle of the Andreev polytope is covered by our bended polygon.
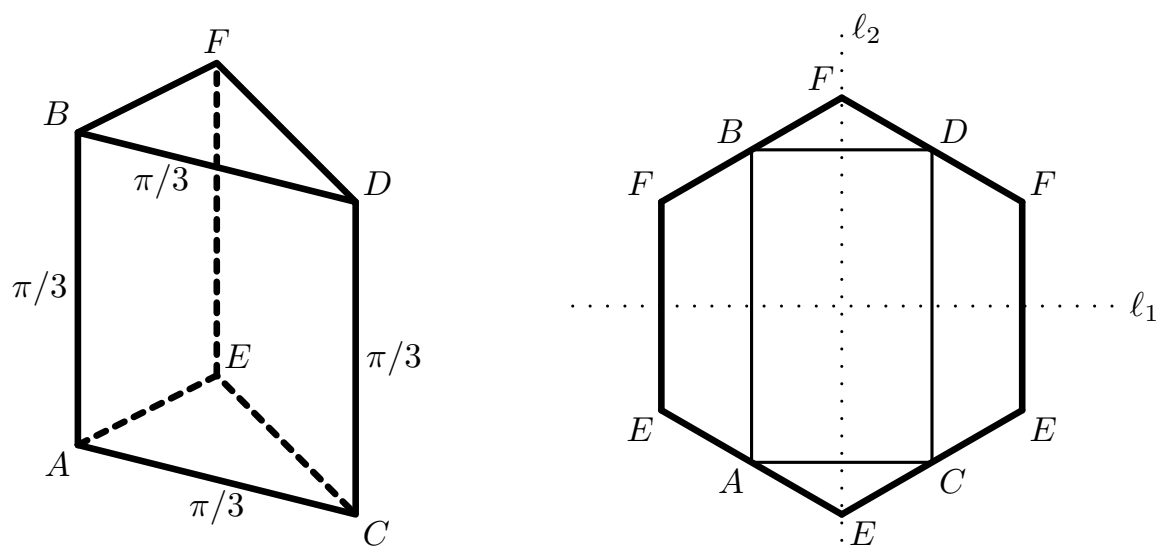

Figure 14. An example of an Andreev polyhedron in $\mathbb{L}^{3}$; we label the dihedral angles $\pi / 3$, all other dihedral angles are $\pi / 2$.

Its development is a (nonregular) 6-gon, whose angles are $\pi / 2$. The lines $\ell_{1}, \ell_{2}$ are axes of symmetry. The polygonal curve $A B D C A$ is a billiard trajectory.

It is easy to reconstruct the lengths of edges of the prism from the combinatorial structure of the development and the billiard trajectory. Indeed, we know the angles of the triangle $A E C$ and of the "trapezoids" $A B F E$, and the equiangular quadrangle $A B D C$. 

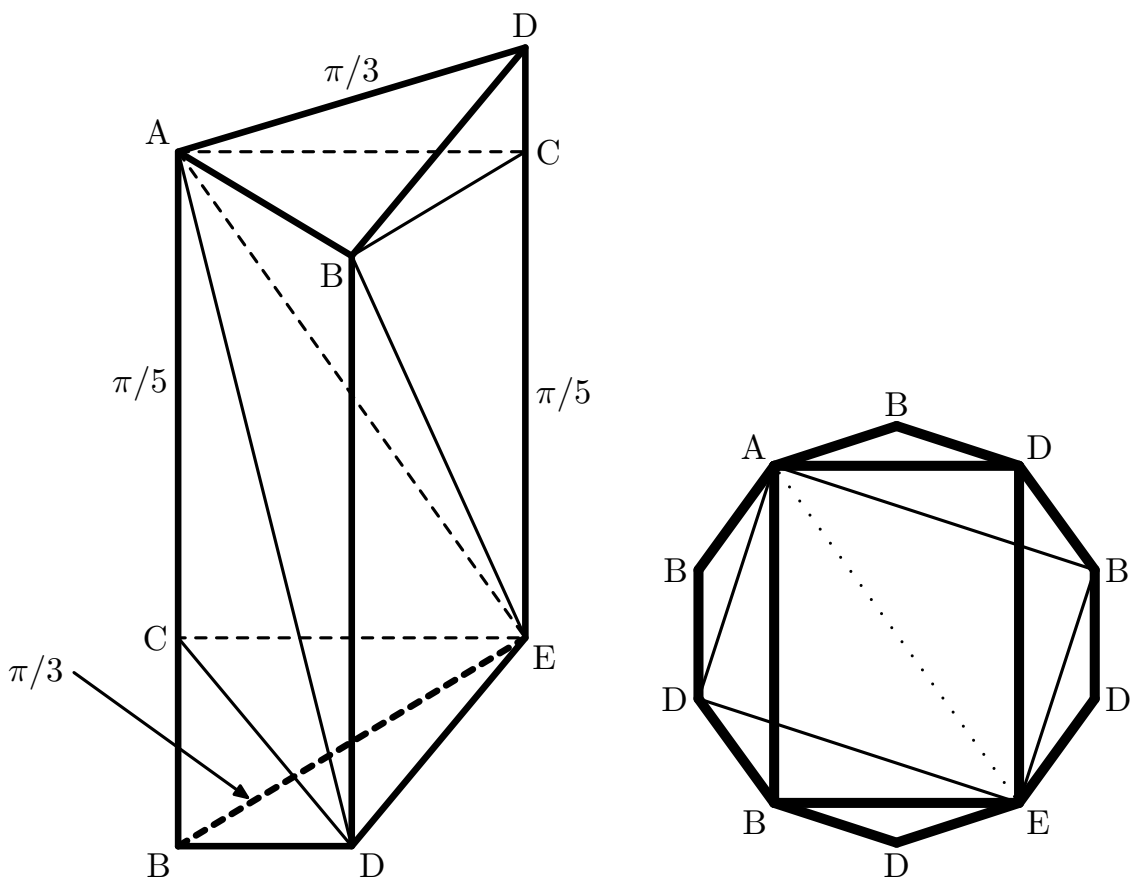

Figure 15. This prism in $\mathbb{L}^{3}$ is a complete development of the Coxeter simplex $A B C D E$ in $\mathbb{L}^{4}$ described in 4.2, It carries all 2dimensional hyperedges of the initial simplex.

The development of the prism is the regular 10-gon with right angles (it also carries all 1-dimensional strata of the 4-dimensional simplex).

Observation 4.2. The surface of an Andreev polyhedron is glued from several bended Coxeter polygons; the rules of bending and the rules of glueing are very rigid.

EXAMPLES of rolling of a 3-dimensional Coxeter polyhedron are given in figures 9 and 14

4.2. Example: Rolling along Andreev polyhedra. Secondly, take a Coxeter polyhedron in $\mathbb{L}^{4}$. Rolling it along 3-dimensional Lobachevsky space, we obtain a Coxeter polyhedron in $\mathbb{L}^{3}$ and also some strange subdivision of this polyhedron.

We present an example. Consider the simplex $\Sigma$ in $\mathbb{L}^{4}$ defined by the Coxeter scheme

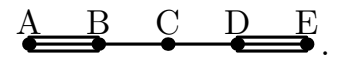

By $A, \ldots, E$ we denote the vertices of the simplex opposite to the corresponding faces. See figure 15 .

Comments to Figure 15. The development of $\Sigma$ is a prism drawn in figure 15. We write labels for the dihedral angles $\neq \pi / 2$. Below a "stratum" means a stratum of the tiling; in particular, the vertical "edge" $A B$ consists of two 1-dimensional strata $B C$ and $C A$ and three 0-dimensional ones, $A, B$, and $C$. 
1) This is a development. Hence any two strata (segments, triangles) having the same notation are equal (for instance $C D=C D, C E=C E, \triangle C B E=\triangle C B E$, etc.).

2) Each stratum (a vertex, a segment) is equipped with a Coxeter group (this group is visible from its dihedral angles)

3) Subdivision of the normal cone $D_{H}$ to a stratum $H$ (a vertex, a segment) is determined by the reduction procedure from subsection 3.3.

For instance, in the vertex $A$ we have the subdivision of the spherical triangle $\mathrm{H}_{3}$ drawn in figure 7 .

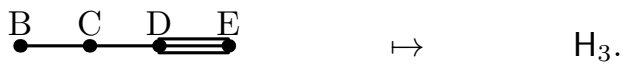

In the normal cone to the edge-stratum $D E$ of the prism, we have the icosahedral subdivision, see figure 8

$$
\stackrel{\mathrm{A}}{\mathrm{B}} \underset{\mathrm{C}}{\mathrm{C}} \quad \mapsto \quad A_{1} \oplus A_{1} .
$$

The normal cone to the segment $A E$ is drawn in figure 4 in particular, both angles of incidence are $\arctan \sqrt{2}$,

$$
\stackrel{\mathrm{B}}{\bullet} \longrightarrow \quad \mapsto \quad A_{1} \oplus \mathrm{R} .
$$

The "front" face $A B D B$ is orthogonal to the sections $C D E$ and $A D E$ (since the lines $C D$ and $A D$ of intersection are equipped with the group $\mathrm{A}_{1} \oplus \mathrm{A}_{1}$ ).

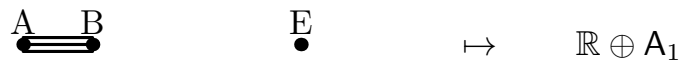

Etc., etc.

4) The prism has two planes of symmetry. This is by chance, partially this is induced by a symmetry of the initial Coxeter scheme 3 . The latter symmetry implies the equality of strata:

$$
A B=D E, \quad A C=C E, \quad A D=B C, \quad B C=C D .
$$

5) Our prism generates a reflection group in $\mathbb{L}^{3}$. The reader can easily imagine a neighborhood of our prism in $\mathbb{L}^{3}$. For instance, near the vertex $A$ we have the picture drawn in figure 8

6) The development of the prism is a regular 10-gon having right angles; the reflection of the "billiard trajectory" $A B E D A$ is of type d) on figure 13. The regularity property follows by reduction from $\mathbb{L}^{4}$, but it is not self-obvious from the picture of the 3-dimensional prism. Obviously, diagonal: $9 B$ are orthogonal to diagonals $D E$ at the points of intersection (see the left side of the figure; but this is not a self-obvious property of this regular 10-gon).

7) We observe the second copy of the polygonal line $A D E B A$ in the development. Bending the 10-gon by this line, we obtain a prism congruent to our prism.

In fact, our 10-gon is the picture on the intersection of two mirrors, denote them by $Y_{1}, Y_{2}$. We can roll the simplex $\Sigma$ along each mirror $Y_{1}, Y_{2}$ and then we roll it again over the intersection $Y_{1} \cap Y_{2}$. We obtain two different pictures on the 10-gon and both are present in the figure 15

${ }^{6}$ There are two diagonals $A B$. 


\section{REFERENCES}

1. Aleksandrov A.D. Convex polyhedra. Gosudarstv. Izdat. Tehn.-Teor. Lit., Moscow, 1950; German translation: Akademie-Verlag, Berlin, 1958; English translation: Springer, 2005

2. Alekseevky, D.V., Kriegl A., Losik M., Michor, P.W., The Riemannian geometry of orbit spaces. The metric, geodesics, and integrable systems, Publ. Math. 62 (2003), no. 3-4, arXiv:math.DG/0102159

3. Alexeevky D.V., Kriegl, A., Losik, M., Michor P.W., Reflection groups on Riemannian manifolds, Annali Mat. pur. appl. 186 (2007), no. 1, 25-58.

4. Alekseevskij, D. V.; Vinberg, E. B.; Solodovnikov, A. S. Geometry of spaces of constant curvature. Geometry, II, 1-138, Encyclopaedia Math. Sci., 29, Springer, Berlin, 1993.

5. Andreev, E. M. Convex polyhedra in Lobachevsky spaces. Mat. Sb. (N.S.) 81 (123) $1970445-$ 478. English transl.: Math. USSR Sb., 10(5), 1970, 413-440

6. Andreev, E. M. Convex polyhedra of finite volume in Lobachevsky space. Mat. Sb. (N.S.) 83 (125) 1970 256-260. English transl. in Math. USSR Sb., 12(3), 225-259 (1971)

7. Andreev, E. M. On intersections of planes bounded an acute angle polyhedron., Math. Notes, 8 (1971) 761-764.

8. Bourbaki, N. Elements de mathematique. Fasc. XXXIV. Groupes et algebres de Lie. Chapitre IV: Groupes de Coxeter et systhemes de Tits. Chapitre V: Groupes engendres par des reflexions. Chapitre VI: systemes de racines. Hermann, Paris 1968.

9. Davis, M. W. Coxeter groups and aspherical manifolds. Algebraic topology, Aarhus 1982 (Aarhus, 1982), 197-221, Lecture Notes in Math., 1051, Springer, Berlin, 1984

10. Davis, M. Groups generated by reflections and aspherical manifolds not covered by Euclidean space. Ann. Math., 117 (1983), 293-324

11. Davis, M. Geometry and topology of Coxeter manifolds. Princeton Univ. Press, 2007.

12. Felikson A., Tumarkin P. On compact hyperbolic Coxeter d-polytops with $(d+4)$ facets. Preprint, available via http://xxx.arHiv.org//math.MG/0510238

13. Khovanskii, A. Combinatorics of sections of polytopes and Coxeter groups in Lobachevsky spaces. The Coxeter legacy, 129-157, Amer. Math. Soc., Providence, RI, 2006.

14. Hodgson, C. D. Deduction of Andreev's theorem from Rivin's characterization of convex hyperbolic polyhedra. Topology '90 (Columbus, OH, 1990), 185-193, Ohio State Univ. Math. Res. Inst. Publ., 1, de Gruyter, Berlin, 1992.

15. Lyusternik, L. A. Convex figures and polyhedra. Translated and adapted from the first Russian edition (1956) by Donald L. Barnett D. C. Heath and Co., Boston, Mass. 1966

16. Rivin, I., Hodgson, C. D.; A characterization of compact convex polyhedra in hyperbolic 3space. Invent. Math. 111 (1993), no. 1, 77-111.

17. Rivin, I. A characterization of ideal polyhedra in hyperbolic 3-space. Ann. of Math. (2) 143 (1996), no. 1, 51-70.

18. Tits, J. Groupes et geometrie de Coxeter, I.H.E.S., 1961, mimeographed notes.

19. Thurston, W., The Geometry and Topology of Three-Manifolds. The text is available via http://www.msri.org/communications/books/gt3m

20. Vinberg, E. B. Discrete linear groups that are generated by reflections. Math. USSR Izvestia 5 (1971), 1083-1119.

21. Vinberg, E.B. Hyperbolic reflection groups, Russian Math. Surveys 40 (1985), 31-75.

22. Vinberg, E. B.; Shvartsman, O. V. Discrete groups of motions of spaces of constant curvature. Geometry, II, 139-248, Encyclopaedia Math. Sci., 29, Springer, Berlin, 1993.

D. V. Alekseevskij: School of Mathematics, Edinburgh University, Edinburgh, EH9 3JZ, UNITED KINGDOM

E-mail address: d.aleksee@ed.ac.uk

P. W. Michor: Fakultät für Mathematik, Universität Wien, Nordbergstrasse 15, A1090 Wien, Austria; and: Erwin Schrödinger Institute of Mathematical Physics, Boltzmanngasse 9, A-1090 Wien, Austria.

E-mail address: Peter.Michor@esi.ac.at 
Yu. A. Neretin: Fakultät für Mathematik, Universität Wien, Nordbergstrasse 15, A-1090 Wien, Austria and Group of Math.Physics, ITEP, B.Cheremushkinskaya, 25, Moscow, 117259, Russia , and MechMath Department, Moscow State University, Vorob'yovy Gory, Moscow, Russia

E-mail address: neretin@mccme.ru 
YuN: 1. I (Yury) use "weird" font to mark my comments

YuN: 2. The title is tentative.

YuN: 3. The Coxeter manifold in the mirror can be disconnected; mirrors can be non-bisecting; for this reason, I minimized the generality. On the other hand, this implies other referees...

\title{
ROLLING OF COXETER POLYHEDRA ALONG MIRRORS
}

\author{
DMITRI V. ALEKSEEVSKI, PETER W. MICHOR, YURII A. NERETIN
}

\begin{abstract}
The topic of the paper are developments of $n$-dimensional Coxeter polyhedra. We show that the surface of such polyhedron admits a canonical cutting such that each piece can be covered by a Coxeter $(n-1)$-dimensional domain.
\end{abstract}

YuN: My text does not contain a direct reference to 2. What to do? On the other hand, I add reference 4 .

\section{Introduction. Coxeter groups}

1.1. Coxeter groups in spaces of constant curvature. Consider a Riemannian space $\mathbb{M}^{n}$ of constant curvature, i.e., a Euclidean space $\mathbb{R}^{n}$, a sphere $\mathbb{S}^{n-1}$, or a Lobachevsky space $\mathbb{L}^{n}$ (on geometry of such spaces, see 4]).

Let $C \subset \mathbb{M}^{n}$ be an intersection of a finite or locally finite collection of halfspaces. 1 .

Consider reflections of $C$ with respect to all $(n-1)$-dimensional faces. Next, consider "new polyhedra" and their reflections with respect to their faces. Etc. The domain $C$ is said to be a Coxeter domain if we get a tilling of the whole space in this way. The group of isometries generated by all such reflections is said to be a reflection group or a Coxeter group (in a narrow sense, see below). We say that a Coxeter group is cocompact if the initial domain $C$ is compact. In this case, we say that $C$ is a Coxeter polyhedron.

Evidently, if $C$ is a Coxeter domain, then the dihedral angles between two neighboring faces of $C$ are of the form $\frac{\pi}{m}$, where $m \geqslant 2$ is an integer. In particular, they are acute, i.e., $\leqslant 90^{\circ}$.

Denote the faces of the polyhedron $C$ by $F_{1}, \ldots, F_{p}$, denote by $s_{1}, \ldots, s_{p}$ the corresponding reflections. Denote by $m_{i j}$ angles between adjacent faces. Evidently,

$$
s_{j}^{2}=1, \quad\left(s_{i} s_{j}\right)^{m_{i j}}=1 .
$$

2000 Mathematics Subject Classification. Primary 51F15, 53C20, 20F55, 22E40.

Key words and phrases. Reflection groups, Coxeter groups, Lobachevsky space, Isometries, Polyhedra, Developments, Trees.

P.W.M. was supported by 'Fonds zur Förderung der wissenschaftlichen Forschung, Projekt P 14195 MAT';

Yu.A.N was supported by Austrian "Fonds zur Förderung der wissenschaftlichen Forschung", project 19064, and also by the Russian Agency on Nuclear Energy, the Dutch fund NWO, grant 047.017.015, and the Japan-Russian grant JSPS-RFBR 07-01-91209.

${ }^{1} \mathrm{~A}$ natural example with an infinite collection of half-spaces is given on Figure 9 
1.2. More terminology. Consider a Coxeter tiling of $\mathbb{M}^{n}$. Below a "chamber" is any ( $n$-dimensional) polyhedron of the tiling. A "face" or "facet" is an $(n-1)$ dimensional face of some chamber; a hyperedge is an $(n-2)$-dimensional edge; a stratum is an arbitrary stratum of codim $\geqslant 1$ of some chamber; a vertex is a vertex.

Also "mirrors" are hyperplanes of reflections. They divide the space $\mathbb{M}^{n}$ into chambers. The group $G$ acts on the set of chambers simply transitively. We denote the reflection with respect to a mirror $Y$ by $s_{Y}$.

Each facet is contained in a unique mirror.

1.3. General Coxeter groups. Take a symmetric $p \times p$ matrix $M$ with positive integer elements, set $m_{j j}=1$; we admit $m_{i j}=\infty$. An abstract Coxeter group is a group with generators $s_{1}, \ldots, s_{n}$ and the relations (11).

For such a group we draw a graph (we use the term "Coxeter scheme") in the following way. Vertices of the graph correspond to generators. We connect $i$ and $j$-th vertices by $\left(m_{i j}-2\right)$ edges. In fact, we draw a multiple edge if $k \leqslant 6$, otherwise we write a number $k$ on the edge.

This rule also assign a graph to each Coxeter polyhedron.

1.4. Spherical Coxeter groups. By definition, a spherical Coxeter group, say $\Gamma$, acts by orthogonal transformations of the Euclidean space $\mathbb{R}^{n+1}$. A group $\Gamma$ is said to be reducible if there exists a proper $\Gamma$-invariant subspace in $\mathbb{R}^{n+1}$. Evidently, the orthogonal complement to a $\Gamma$-invariant subspace is $\Gamma$-invariant.

The classification of irreducible Coxeter groups is well known'2, see Bourbaki 8. The list consists of Weyl groups of semisimple Lie algebras (= Killing's list of root systems) + dihedral groups + groups of symmetries of the icosahedron and 4-dimensional hypericosahedron (the table is given Section 3).

This also gives a classification of reducible groups.

1.5. Coxeter equipments. Next, consider an arbitrary Coxeter polyhedron in $\mathbb{R}^{n}, \mathbb{S}^{n}$, or $\mathbb{L}^{n}$. Consider a stratum $H$ of codimension $k$, it is an intersection of $k$ faces, $H=F_{i_{1}} \cap \cdots \cap F_{i_{k}}$. The reflections with respect to the faces $F_{i_{1}}, \ldots, F_{i_{k}}$ generate a Coxeter group, denote it by $\Gamma(H)=\Gamma\left(F_{i_{1}}, \ldots, F_{i_{k}}\right)$.

This group is a transparent object. Consider the orthocomplement to the stratum $H$ and the sphere surrounding the stratum in this orthocomplement. Then $\Gamma(H)$ is a reflection group of this sphere.

If $H \subset H^{\prime}$, then we have the tautological embedding

$$
\iota_{H^{\prime}, H}: \Gamma\left(H^{\prime}\right) \rightarrow \Gamma(H) .
$$

If $H \subset H^{\prime} \subset H^{\prime \prime}$, then

$$
\iota_{H^{\prime \prime}, H}=\iota_{H^{\prime}, H^{\prime} \iota_{H^{\prime \prime}, H^{\prime}}}
$$

Such a collection of groups and homomorphisms is said to be a Coxeter equipment.

1.6. Cocompact Euclidean Coxeter groups. Here classification is also simple and well known, see Bourbaki 8 . Any such group $\Gamma$ contains a normal subgroup $\mathbb{Z}^{n}$ acting by translations and $\Gamma / \mathbb{Z}^{n}$ is a spherical Coxeter group.

\footnotetext{
${ }^{2}$ Actually, these objects were known to Ludwig Schläfli and Wilhelm Killing in XIX century. In 1924, Hermann Weyl identified these groups as reflection groups, in 1934 Harold Coxeter gave a formal classification and also classified Euclidean groups.
} 
1.7. Coxeter groups in Lobachevsky spaces. For Lobachevsky spaces the situation drastically differs.

a) Coxeter polygons on Lobachevsky plane are arbitrary $k$-gons with angles of the form $\pi / m_{j}$. The sum of exterior angles must satisfy $\sum\left(\pi-\pi / m_{j}\right)>2 \pi$. If $k>5$ this condition holds automatically. For $k=4$ this excludes rectangles, also few triangles are forbidden (in fact, spherical and Euclidean triangles). A Coxeter $k$-gon with prescribed angles depends on $(k-3)$ parameters.

b) In dimensions $n>2$ Coxeter polyhedra are rigid. There is a lot of Coxeter groups in spaces of small dimensions $(n=3,4,5)$, but for $n \geqslant 30$ there is no Coxeter groups with compact fundamental polyhedron at all, see Vinberg [21, Vinberg, Shvartsman, 22]. For $n>996$ there is no Coxeter groups of finite covolume (Prokhorov, Khovanskii, 1986, see 13]); the maximal dimensions of known examples are: 8 for compact polyhedra (Bugaenko), and 21 for a polyhedron of finite volume (Borcherds). For $n=3$ there is a nice Andreev's description [5] of all Coxeter polyhedra, it is given in the following two subsections.

1.8. Acute angle polyhedra in $\mathbb{L}^{3}$. First, we recall the famous (and highly nontrivial) Steinitz Theorem (see, e.g., 15]) about possible combinatorial structure of convex polyhedra in $\mathbb{R}^{3}$.

Since the boundary of a polyhedron is a topological sphere $S^{2}$, edges form a connected graph on the sphere, it divides the sphere into polygonal domain (we use the term 'face' for such a domain). There are the following evident properties of the graph:

- each edge is contained in 2 faces;

- each face has $\geqslant 3$ vertices;

- the intersection of any pair of faces can by the empty set, a vertex, or an edge.

Theorem. (Ernst Steinitz) Any graph on the sphere $S^{2}$ satisfying the above conditions can be realized as a graph of edges of a convex polyhedron.

Our next question is the existence of a convex polyhedron in $\mathbb{L}^{3}$ of a given combinatorial structure with a given acute dihedral angles ('acute' means $\leqslant \pi / 2$; may be the term 'non-obtuse' is better). There are the following a priori properties of such polyhedra:

1) Evidently, all spacial angles are simplicial; equivalently each vertex of the graph is contained in 3 edges. The angles $\varphi_{1}, \varphi_{2}, \varphi_{3}$ in a given vertex satisfy

$$
\varphi_{1}+\varphi_{2}+\varphi_{3}>2 \pi \text {. }
$$

2) Dihedral angles determine angles in each face (by the spherical cosine theorem). A face must be a Lobachevsky polygon, i.e. the sum of its exterior angles must be $\geqslant 2 \pi$. Since dihedral angles are acute, face angles also are acute; therefore our conditions forbid only rectangles and some triangles.

3) The last restriction is non-obvious. We say that a $k$-prismatic element of a convex polyhedron $C$ is a collection sequence

$$
F_{1}, \quad F_{2}, \quad \ldots, \quad F_{k}, \quad F_{k+1}:=F_{1}
$$

of faces such that $F_{k}$ and $F_{k+1}$ have a common edge, and all triple intersections $F_{i} \cap F_{j} \cap F_{k}$ are empty. 


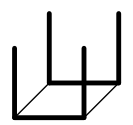

Figure 1. The following configuration with dihedral angles $=\pi / 2$ on thick edges is forbidden in Andreev Theorem. In this case, we get a quadrangle with right angles, such quadrangles do not exist.
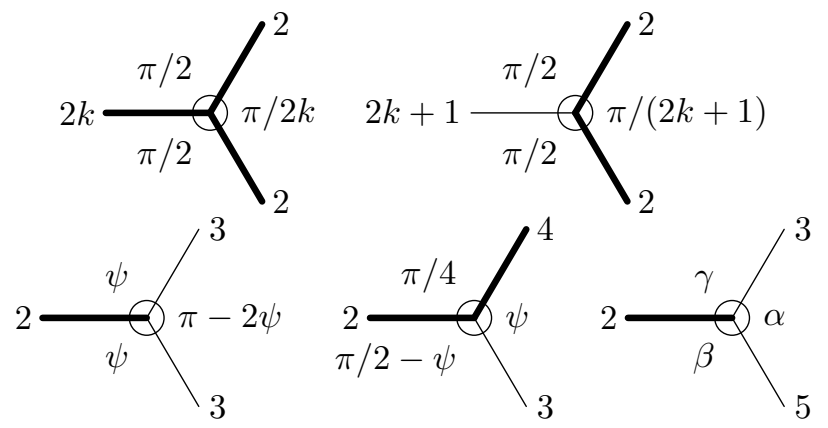

Figure 2. We draw all possible types of vertices of Andreev polyhedra. We present labels $m_{j}$ on edges and flat angles in faces. Here $\psi=\arctan \sqrt{2}$ and $\alpha, \beta, \gamma$ are explicit angles with $\alpha+\beta+\gamma=\pi / 2$.

Evaluations of angles are given in figures in Section 2

We draw a thick line iff the label is even.

Lemma. (Andreev) For any prismatic element in an acute angle polyhedron, the sum of exterior dihedral angles is $>2 \pi$.

Theorem. (Andreev) Consider a Steinitz-admissible 3-valent spherical graph

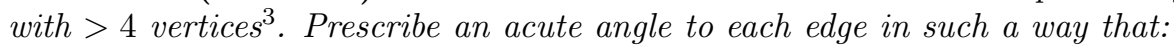

- the inequality (2) in each vertex is satisfied;

- all 3-and 4-prismatic elements satisfy the previous lemma;

- we forbid the configuration given on Figure 1.

Under these exceptions, there exists a unique convex polyhedron $\subset \mathbb{L}^{3}$ of the given combinatorial structure and with the given acute angles.

The uniqueness is a rigidity theorem of Cauchy type (see [1, [15]). The existence is a deep unusual fact; it is a special case of the theorem of Aleksandrov type (see 1]) obtained later by Rivin, see [16], 14]

For some applications of Andreev and Rivin Theorems to elementary geometry, see Thurston [19, Rivin [17.

1.9. Andreev polyhedra. Andreev Theorem provides us a description of all Coxeter polyhedra in $\mathbb{L}^{3}$. Now angles have the form $\pi / m_{i j}$ with integer $m_{i j}>1$. Now, we simply write labels $m_{i j}$ on edges in admissible way. $\mathbb{L}^{3}$.

Below the term "Andreev polyhedron" means a compact Coxeter polyhedron in

All possible pictures at vertices of Andreev polyhedra are given in Figure 2

\footnotetext{
${ }^{3}$ Simplices are exceptions. However, their examination is simple, Lanner, 1950, see, e.g., 22.
} 
1.10. Results of the paper. Consider a convex polyhedron $C$ in a space $\mathbb{M}^{n}$ of constant curvature. Following Alexandrov [1, we regard the boundary $\Xi=\partial C$ of $C$ as an $(n-1)$-dimensional manifold of constant curvature with singularities. In the case $n=3$, we get a two-dimensional surface with conic singularities of negative curvature (see e.g. Figure 2 in all the cases the sum of angles at a singularity is $<2 \pi)$.

Now, cut $\Xi$ along hyperdges with even labels (i.e., hyperedges with dihedral angles $\pi / 2 k)$. Let $\Omega_{1}, \Omega_{2}, \ldots$ be connected pieces of the slit YuN: slit? surface.

Theorem 1.1. Universal coverings $\Omega_{j}^{\sim}$ of all $\Omega_{j}$ are Coxeter domains in $\mathbb{M}^{n-1}$.

Proof for Andreev polyhedra. We simply look to Figure 2 In all the cases, angles between thick edges are Coxeter...

We also describe tilings of mirrors, groups of transformations of mirrors induced by the initial Coxeter group (Theorem 2.10) and the Coxeter equipments of $\Omega_{j}^{\sim}$ (Theorem 3.2).

Addendum to the paper contains two examples of 'calculation' of developments, for an Andreev prism $\subset \mathbb{L}^{3}$ and for a Coxeter simplex $\subset \mathbb{L}^{4}$. The proof of the Andreev Theorem is nonconstructive. In various explicit cases, our argumentation allows to construct of Andreev polyhedra from the a priori information about its development. Our example illustrates this phenomenon.

On the other hand, there arises a natural problem of elementary geometry:

- Which Andreev polyhedra are partial developments of 4-dimensional Coxeter polyhedra? Is it possible to describe all 3-dimensional polyhedra that are faces of 4-dimensional Coxeter polyhedra?

Our main argument (Rolling Lemma 2.1) is very simple, it is valid in a wider generality, we briefly discuss such possibilities in next two subsections.

\subsection{Polyhedral complexes and projective Coxeter polyhedra.}

Theorem. (Tits) Any Coxeter group can be realized as a group generated by a collection of reflections $s_{1}, \ldots, s_{p}$ with respect to hyperplanes in an open convex subset in a real projective space $\mathbb{R P}^{n}$. The closure of a chamber is a convex polyhedron.

See also Vinberg 20.

The formal construction is simple (but it requires a substantiation), we take a collection of unit vectors $e_{1}, \ldots, e_{p}$ in a pseudo-Euclidean space such that

$$
\left\langle e_{i}, e_{j}\right\rangle=\cos \frac{\pi}{m_{i j}} \quad \text { if } m_{i j}<\infty \text { and }\left\langle e_{i}, e_{j}\right\rangle \geqslant 1 \text { otherwise }
$$

A chamber is determined by the inequalities $\left\langle v, e_{j}\right\rangle>0$

1.12. Wider generalities. Nikolas Bourbak 1 proposed a way to build topological spaces from YuN: from? Coxeter groups. M. Davis used this approach in numerous papers (see e.g. [9, [10]) and the book [11; in particular he constructed nice examples/counterexamples in topology.

Also it is possible to consider arbitrary Riemannian manifolds equipped with a discrete isometric action of a Coxeter group such that the set of fixed points of each generator is a totally geodesic submanifold and the generators act as reflections with

\footnotetext{
${ }^{4}$ Apparently, he used the work by Jacque Tits [18; the latter text is inaccessible for the authors.
} 

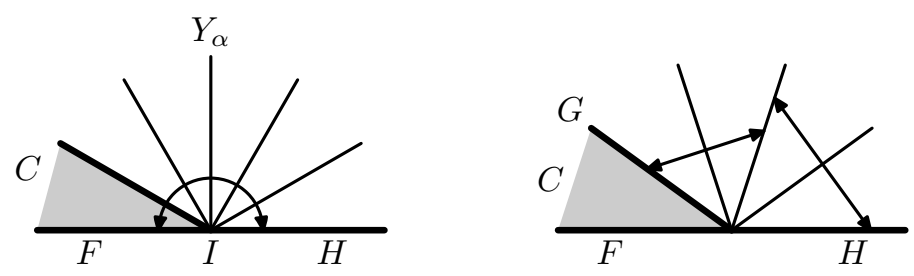

Figure 3. Even and odd labels. Proof of Rolling Lemma.

respect to these submanifolds. In this context, a chamber itself can be topologically non-trivial object, see [11, 3].

\section{Rolling OF CHAMBeR}

In this section, $\mathbb{M}^{n}$ is a space $\mathbb{L}^{n}, \mathbb{S}^{n}, \mathbb{R}^{n}$ of constant curvature equipped with a Coxeter group $\Gamma$ or, more generally, any space described in Subsection 1.11.

Fix a mirror $\mathbb{X}^{n-1}$ in $\mathbb{M}^{n}$. Consider intersections of $\mathbb{X}^{n-1}$ with other mirrors $Y_{\alpha}$. The set $\mathbb{X}^{n-1} \backslash \cup Y_{\alpha}$ is a disjoint union of open facets. Thus, we get a tiling of $\mathbb{X}^{n-1}$ by facets.

Our aim is to describe this tiling in the terms of geometry of a chamber.

\subsection{Rolling lemma.}

Lemma 2.1. Let $I \subset \mathbb{X}^{n-1}$ be an $(n-2)$-dimensional hyper-edge of our tiling. Let $F, H \subset \mathbb{X}^{n-1}$ be the facets adjacent to $I$.

a) If the label $m_{I}$ of $I$ is even, then $I$ is contained in a certain mirror $Y_{\alpha}$ orthogonal to $X$. In particular $s_{Y_{\alpha}} F=H$.

b) Let the label $m$ be odd. Let $C$ be a chamber adjacent to the facet $F$. Let $G$ be another facet of $C$ adjacent to the same hyper-edge $I$. Then $G$ is isometric to $H$. More precisely, there is $\gamma \in \Gamma$ fixing all the points of I such that $\gamma G=H$.

Proof is given in Fig 3

2.2. Algorithm generating the tiling. Let $C$ be a chamber adjacent to a facet $F \subset \mathbb{X}^{n-1}$. Consider an hyper-edge $I$ of $C$ lying in $\mathbb{X}^{n-1}$.

Operation 1. Let the hyper-edge $I$ be odd. Consider a facet $G \neq F$ of $C$ adjacent to $I$, consider the corresponding $\gamma$ from Lemma 2.1 and draw $\gamma G$ on $\mathbb{X}^{n-1}$

Operation 2. If the hyper-edge $I$ is even, then we reflect $F$ in $\mathbb{X}^{n-1}$ with respect to $I$.

We perform all the possible finite sequences of such operations. By the Rolling Lemma, we get the whole tiling of the mirror $\mathbb{X}^{n-1}$.

REMARK. Let $\mathbb{M}^{n}=\mathbb{R}^{3}, \mathbb{S}^{3}, \mathbb{L}^{3}$ be a usual 3-dimensional space of constant curvature. Operation 1 corresponds to rolling of a polyhedron $C$ along the hyperplane $\mathbb{X}^{n-1} \sim \mathbb{M}^{2}$ over an edge. 

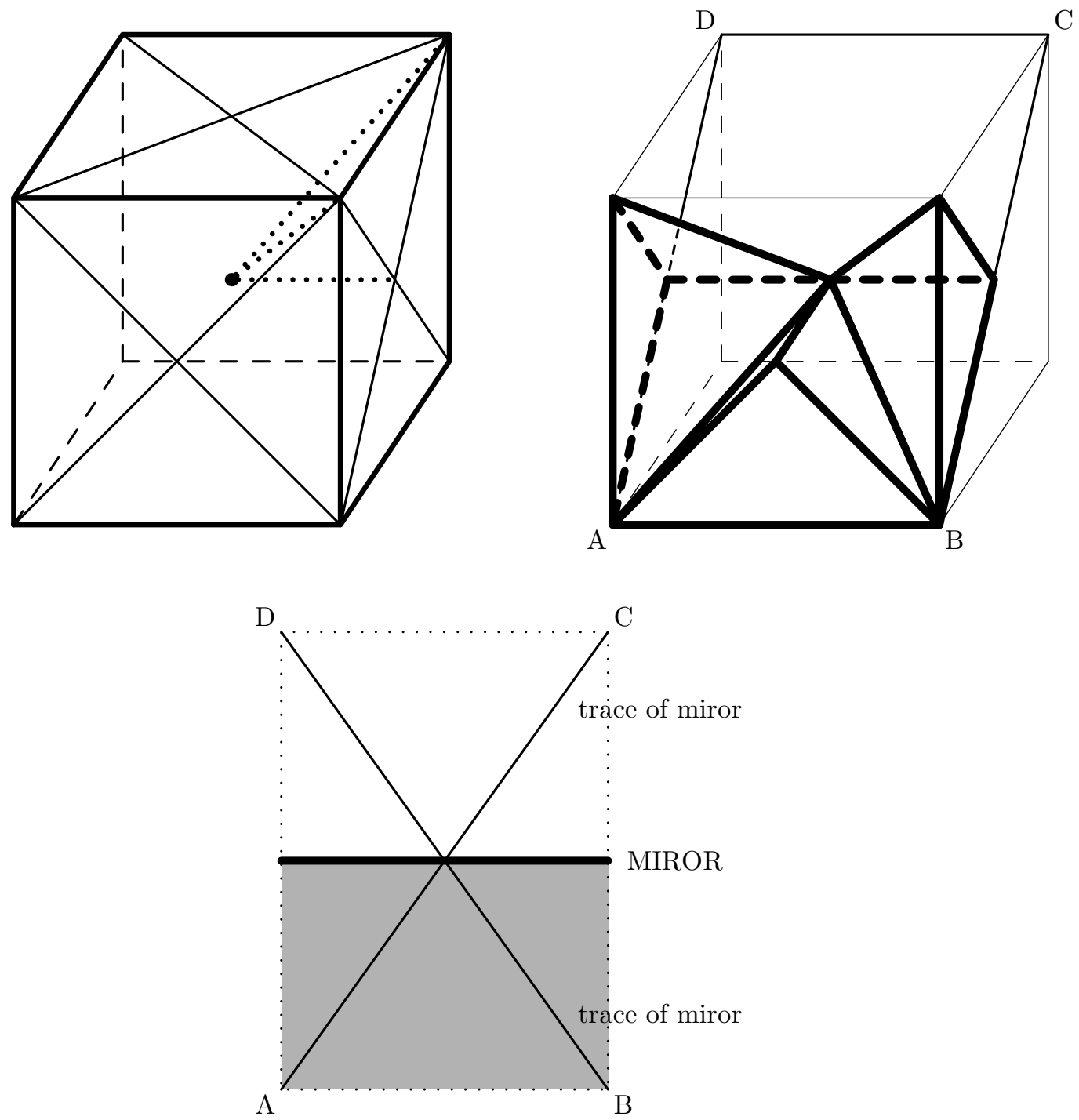

FiguRE 4. Example of rolling: the reflection group $A_{3}$ in $\mathbb{R}^{3}$. The mirrors are planes passing through opposite edges of the cube. There are 24 Weyl chambers, which are simplicial cones with dihedral angles $\pi / 3, \pi / 3, \pi / 2$ (we draw them as simplices). Rolling of a Weyl chamber by the mirror $A B C D$ produces a half-plane.

We can also regard $A_{3}$ as a reflection group on the 2-dimensional sphere $\mathbb{S}^{2}$.

2.3. The group preserving the mirror $\mathbb{X}^{n-1}$. For a mirror $\mathbb{X}^{n-1}$, consider the group $\Gamma_{*}=\Gamma_{*}\left(\mathbb{X}^{n-1}\right)$ of all the isometries of $\mathbb{X}^{n-1}$ induced by elements of $\Gamma$ preserving $\mathbb{X}^{n-1}$. 


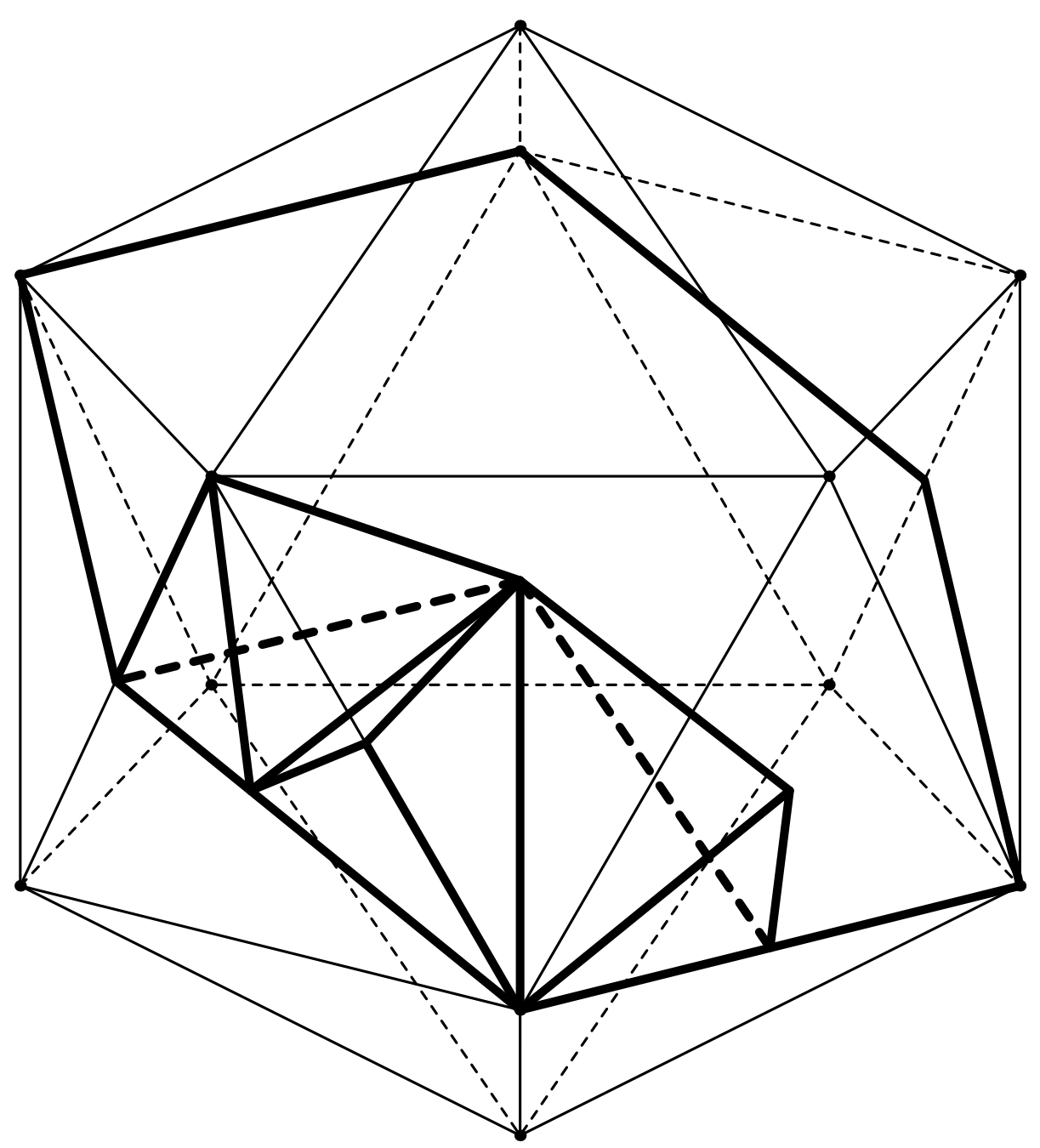

FIgURE 5. Example of rolling: the icosahedral group $\mathrm{H}_{3}$. It is generated by reflections with respect to bisectors of segments connecting midpoints of opposite edges of the icosahedron. The bisectors separate $\mathbb{R}^{3}$ into 120 simplicial cones with dihedral angles $\pi / 2, \pi / 3, \pi / 5$. In the figure the simplicial cones are cut by the surface of the icosahedron.

We show an admissible rolling of a Weyl chamber along a mirror. The final chamber in the mirror is a quadrant.

If $\gamma \in \Gamma$ preserves $\mathbb{X}^{n-1}$, then $s_{\mathbb{X}^{n-1}} \gamma$ also preserves $\mathbb{X}^{n-1}$. Thus each element of $\Gamma_{*}$ is induced by two different elements of $\Gamma$.

Observation 2.2. Let $F_{1}, F_{2} \subset \mathbb{X}^{n-1}$ be equivalent facets. There is a unique element $\mu \in \Gamma_{*}\left(\mathbb{X}^{n-1}\right)$ such that $\mu F_{1}=F_{2}$. 

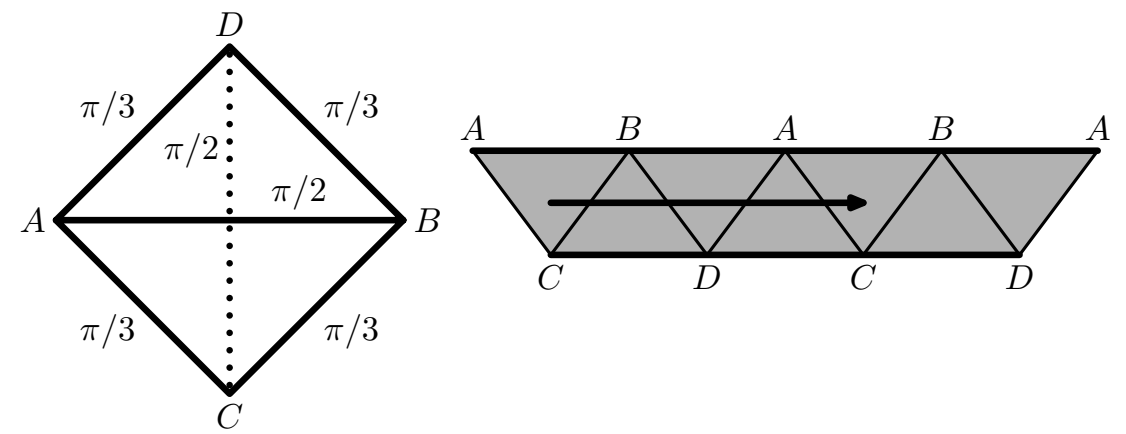

Figure 6. Example of rolling: the (affine) Euclidean reflection group $\widetilde{A}_{4}$ in $\mathbb{R}^{3}$. A chamber is the simplex $A B C D$. Rolling through $A B$ and $C D$ is forbidden. Deleting these edges from the surface of the simplex, we get a non-simply connected surface. Hence, the process of rolling is infinite. The arrow shows the deck transformation induced by the generator of the fundamental group.
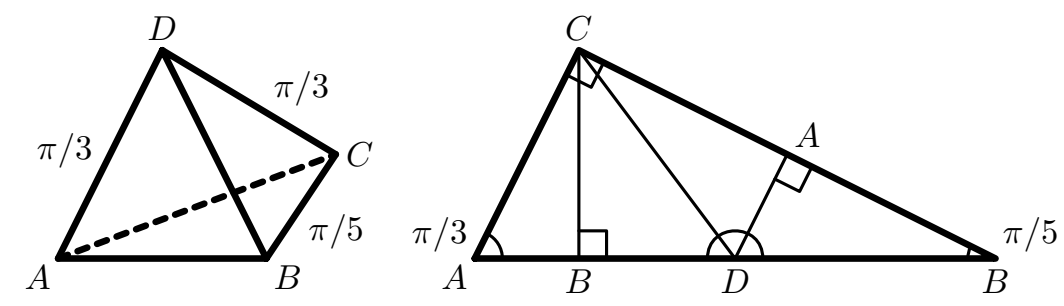

FiguRE 7. Example of rolling: the hypericosahedral group $\mathrm{H}_{4}$ acting on the 3 -dimensional sphere $\mathbb{S}^{3}$. The chamber is is the spherical simplex drawn in the figure (we omit all labels $\pi / 2$ on edges).

The angle $=\pi$ on the development at $D$ was evaluated in Fig 4 The right angle at $C$ was evaluated in Fig 5

The spherical triangle $A B C$ is present on the circumscribed sphere in the next figure (in spite of the absence of the sphere itself).

2.4. Reflections in mirrors and the new chamber. Consider all the mirrors $Z_{\alpha} \subset \mathbb{M}^{n}$ orthogonal to our mirror $\mathbb{X}^{n-1}$. The corresponding reflections $s_{Z_{\alpha}}$ generate a reflection group on $\mathbb{X}^{n-1}$; denote this group by $\Delta=\Delta\left(\mathbb{X}^{n-1}\right)$.

Observation 2.3. $\Delta$ is a normal subgroup in $\Gamma_{*}\left(\mathbb{X}^{n-1}\right)$.

Indeed, if $s$ is a reflection, then $\gamma^{-1} s \gamma$ is a reflection.

Consider a chamber $C$ of $\mathbb{M}^{n}$ lying on YuN: Is Iying on OK? this can mean "contained"? $\mathbb{X}^{n-1}$ (i.e., having a facet in $\mathbb{X}^{n-1}$ ) and consider all possible sequences of admissible rollings, i.e., we allow Operation 1 of Algorithm and forbid Operation 2. Denote by $B \subset \mathbb{X}^{n-1}$ the domain obtained by rolling, tiled as described.

Theorem 2.4. $B$ is a chamber of the reflection group $\Delta\left(\mathbb{X}^{n-1}\right)$. 


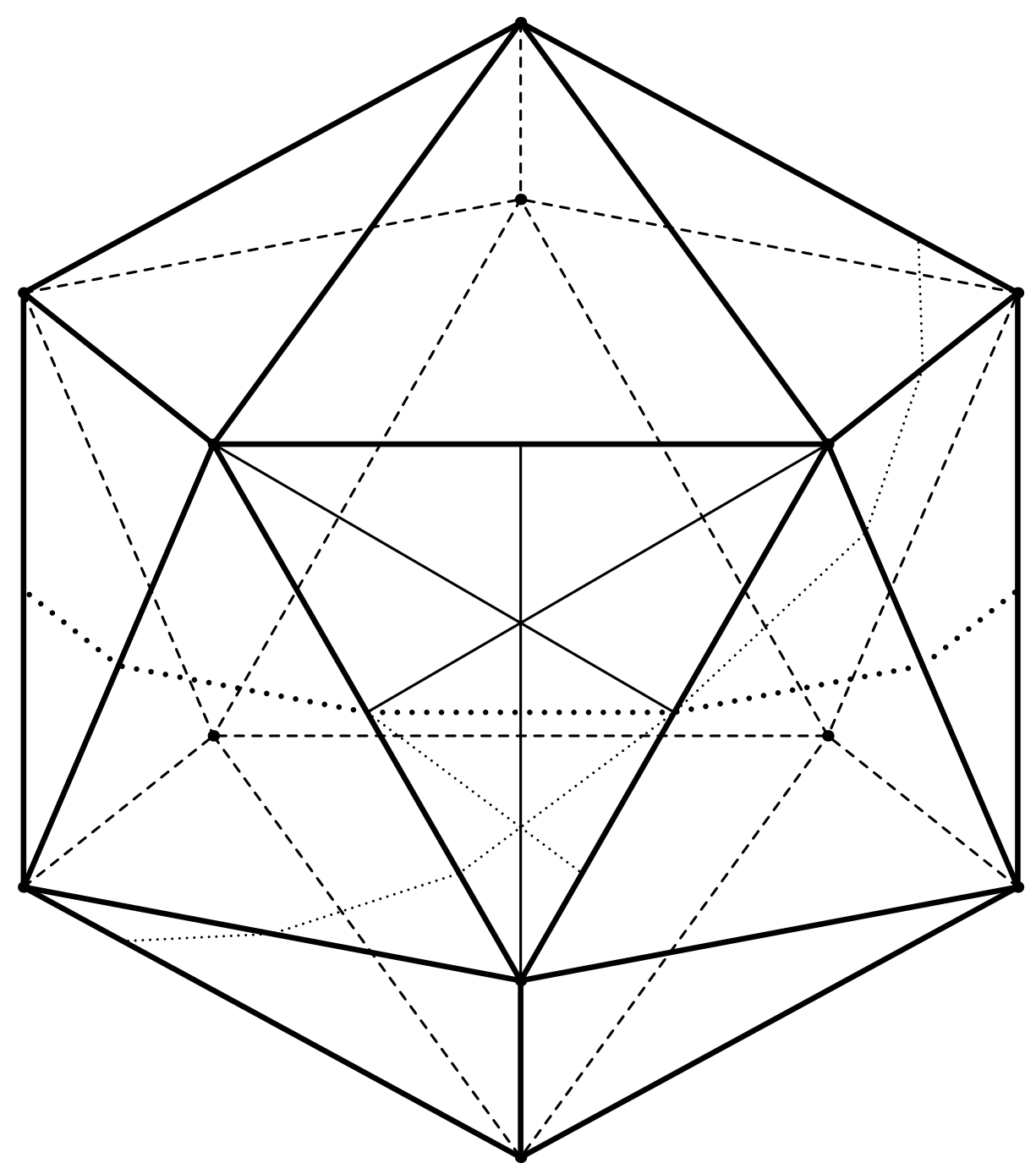

FiguRE 8. Example of rolling: the hypericosahedral group $\mathrm{H}_{4}$ acting in $\mathbb{R}^{4}$. The figure presents the tiling of a mirror, i.e., of $\mathbb{R}^{3}$, by simplicial cones. We draw intersections of simplicial cones with the boundary of the icosahedron. Consider 3 types of 'axes' of the icosahedron:

A) segments connecting midpoints of opposite edges;

B) segments connecting central points of opposite faces;

C) diagonals connecting opposite vertices.

Consider bisectors of all such segments. Type A bisectors are mirrors. They divide $\mathbb{R}^{3}$ into 120 simplicial chambers. Six chambers are presented in the front face of the icosahedron.

Adding bisectors of type $\mathrm{B}$ and $\mathrm{C}$ we obtain a partition of $\mathbb{R}^{3}$ into 480 simplicial cones. This is the desired tiling.

In this figure, we present subdivisions of two chambers. A proof of this picture is contained on Fig[ 

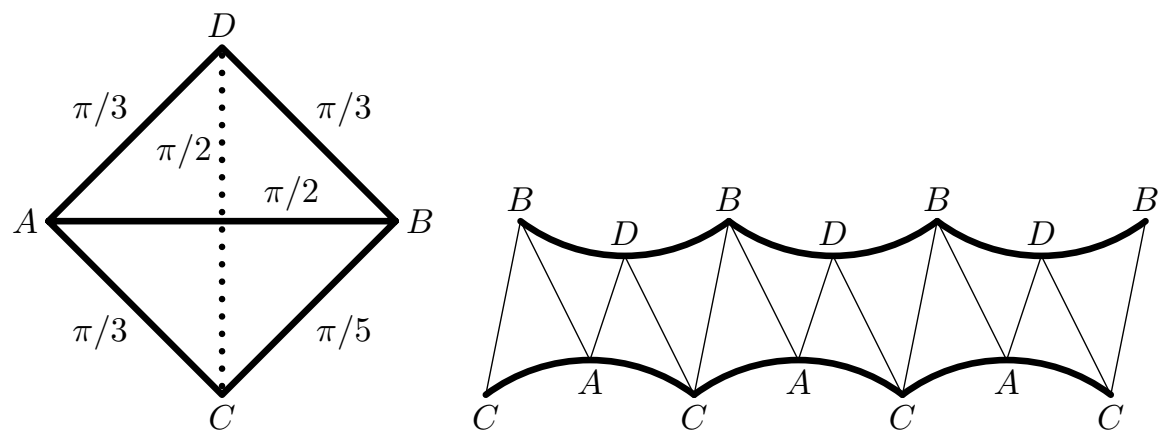

Figure 9. Example. A Coxeter simplex in $\mathbb{L}^{3}$. Its development is an infinite 'strip' $\subset \mathbb{L}^{2}$ bounded by two infinite polygonal curves, interior angles between segments of polygonal curves are $\pi / 2$ and $\pi$.

Proof. We can not roll further if and only if we meet a "vertical" mirror.

EXAMPLES OF ROLLING. Some examples of rolling corresponding to the usual spherical Coxeter groups

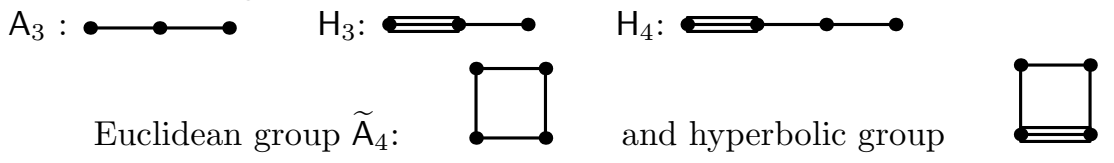

are given on Fig 49] In these figures, we also evaluate the new chamber $B$.

Lemma 2.5. Each $(n-3)$-dimensional stratum of our tiling of $\mathbb{X}^{n-1}$ is contained in a mirror of the group $\Delta\left(\mathbb{X}^{n-1}\right)$.

Proof. This stratum is equipped with a finite 3-dimensional Coxeter group (i.e., $A_{3}, B_{3}, H_{3}, A_{1} \oplus G_{2}^{m}, A_{1} \oplus A_{1} \oplus A_{1}$, see Table below). For each mirror of such a group there exists an orthogonal mirror.

2.5. Rolling scheme. Denote by $\Xi(C)$ the surface of the initial chamber $C$, let $\Xi^{\prime}(C)$ be the surface with all even edges deleted.

Lemma 2.6. $\Xi^{\prime}$ does not contain $(n-3)$-dimensional strata of $C$.

This is rephrasing of Lemma 2.5

Consider the graph, whose vertices are the facets of $\Xi^{\prime}$; vertices are connected by an edge if the corresponding facets are neighbors in $\Xi^{\prime}$. We call this graph the Rolling scheme. In fact, the Rolling scheme is the Coxeter scheme with removed even (and infinite) edges.

Proposition 2.7. The surface $\Xi^{\prime}$ is homotopically equivalent to the Rolling scheme.

2.6. Proof of Proposition 2.7, Let $U$ be a convex polyhedron in $\mathbb{R}^{n}$, denote by $\Xi$ its surface. Choose a point $A_{j}$ in interior of each $(n-1)$-dimensional face. Choose a point $B_{k}$ in interior of each $(l-2)$-dimensional boundary stratum (hyperedge) of $U$.

Draw the segment $\left[A_{j}, B_{k}\right]$ iff the face contains the hyperedge. Thus we get a graph $T$ on the surface of the polyhedron $C$ whose vertices are enumerated by faces 
a)

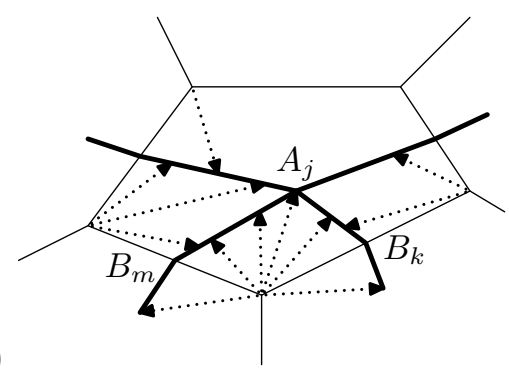

b)

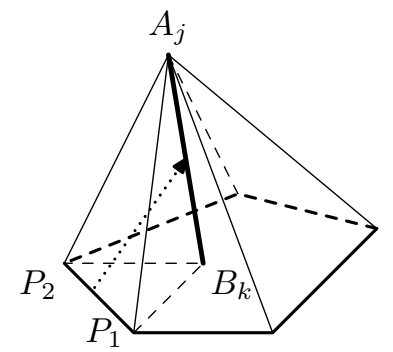

Figure 10. Proof of Lemma 2.8

a) $n=3$. Graph on a surface of a 3 -dimensional polytop and a retraction. Recall that we have removed vertices.

b) $n=4$. A piece of a 3 -face of 4-dimensional polyhedron. Recall that 1-dimensional edges are removed. Inside a simplex $P_{1} P_{1} A_{j} B_{k}$ the retraction is the projection to $A_{j} B_{k}$ with center on the segment $P_{1} P_{2}$. Note that all segments connecting $A_{j} B_{k}$ and $P_{1} P_{2}$ are pairwise non-intersecting.

of $U$ and edges are enumerated by hyperedges of $U$. Denote by $\Xi \nabla$ the surface of the polyhedron $S$ without boundary strata of dimension $(n-3)$.

Lemma 2.8. The graph $T$ is a deformation retract of $\Xi \nabla$. Moreover, it is possible to choose a homotopy that preserves all faces and all hyperedges.

Proof. See Fig. 10

Proposition 2.7 follows from Lemma 2.8

YuN: Under percents there is a non-edited proof of Lemma

2.7. Action of the fundamental group on mirror. Again, let $F$ be a facet in $\mathbb{X}^{n-1}$, let $C$ be a chamber of $\mathbb{M}^{n}$ lying on $F$, let $B \supset F$ be the chamber of the reflection group $\Delta\left(\mathbb{X}^{n-1}\right)$ obtained by rolling $C$, as described in Subsection 2.4

Let $\Omega$ be a connected component of $\Xi^{\prime}$ containing the facet $F$.

Let $F_{1}, \ldots, F_{r}$ be facets $\subset \Omega$. We can think that each facet has its own color; thus the mirror $\mathbb{X}^{n-1}$ is painted in $r$ colors. Moreover, for each facet $H \in \mathbb{X}^{n-1}$ there is a canonical bijection ('parametrization') from the corresponding $F_{i} \subset \Omega$ to $H$. We say that a bijection $\mathbb{X}^{n-1} \rightarrow \mathbb{X}^{n-1}$ (or $B \rightarrow B$ ) is an isomorphism if it preserves the coloring and commutes with the parameterizations.

Proposition 2.9. a) The chamber $B \subset \mathbb{X}^{n-1}$ is the universal covering of $\Omega$.

b) Any deck transformations of $B$ is an isomorphism $B \rightarrow B$ and admits a unique extension to an isomorphism of the mirror $\mathbb{X}^{n-1}$.

c) Each isomorphism $\mu \in \Gamma_{*}\left(\mathbb{X}^{n-1}\right)$ preserving $B$ is induced by a deck transformation.

Proof. a) Denote by $\Omega^{\sim}$ the universal covering of $\Omega$. The chamber $B$ was constructed as the image of $\Omega^{\sim}$. Moreover, the map $\Omega^{\sim} \rightarrow C$ is locally bijective. On the other hand, a chamber on a simply connected manifold is simply connected see (see [3], 2.14); therefore $B \simeq \Omega^{\sim}$. 


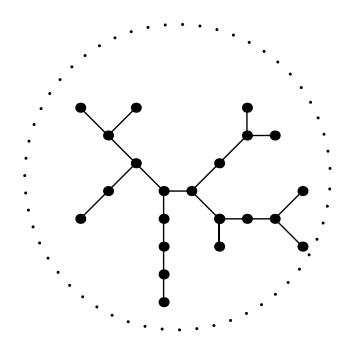

FiguRE 11. A graph of vicinity of $(n-1)$-dimensional facets in the new $(n-1)$-dimensional chamber $B$ is a tree.

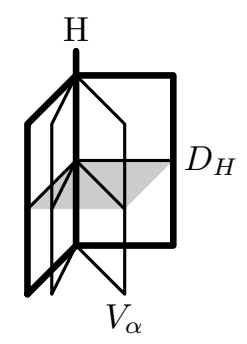

FiguRE 12. Subdivision of the cone normal to a stratum.

b) A deck transformation $B \rightarrow B$ is an isometry by rolling rules. Let a deck transformation send a facet $F$ to $F^{\prime}$. Then the facets $F, F^{\prime}$ are $\Gamma$-equivalent, the corresponding map is an isometry of $\mathbb{X}^{n-1}$.

c) Let $F \subset \mathbb{X}^{n-1}$ be a facet. We take the deck transformation sending $F$ to $F^{\prime}$.

\subsection{Description of $\Gamma_{*}\left(\mathbb{X}^{n-1}\right)$.}

Theorem 2.10. The group $\Gamma_{*}\left(\mathbb{X}^{n-1}\right)$ is a semidirect product $\operatorname{Deck}(B) \ltimes \Delta\left(\mathbb{X}^{n-1}\right)$.

Proof. Indeed, the group $\Delta\left(\mathbb{X}^{n-1}\right)$ acts simply transitively on the set of chambers in $\mathbb{X}^{n-1}$; the group $\operatorname{Deck}(B)$ acts simply transitively on the set of facets of a given type in the chamber $B$.

\section{REDUCTION OF EQUIPMENT}

Keep the notation of the previous section. Our aim is to describe the Coxeter equipment of new chamber $B$.

3.1. Combinatorial structure of the tiling of the chamber. Consider a graph $\mathfrak{F}$ whose vertices are enumerated by $(n-1)$-facets lying in $B$, two vertices are adjoint YuN: connected with? with? to one edge if they have a common $(n-2)$-dimensional stratum (a former hyperedge in $\mathbb{M}^{n}$ ).

Observation 3.1. $\mathfrak{F}$ is a tree.

Proof. Indeed, the universal covering over a graph is a tree.

If the initial Rolling scheme is a tree, then we get the same tree. If the Rolling scheme contains a cycle, then we get an infinite tree (examples: Figures 6 , 9 the Rolling schemes contain 1 cycle). 
Table. Reduction of spherical Coxeter schemes

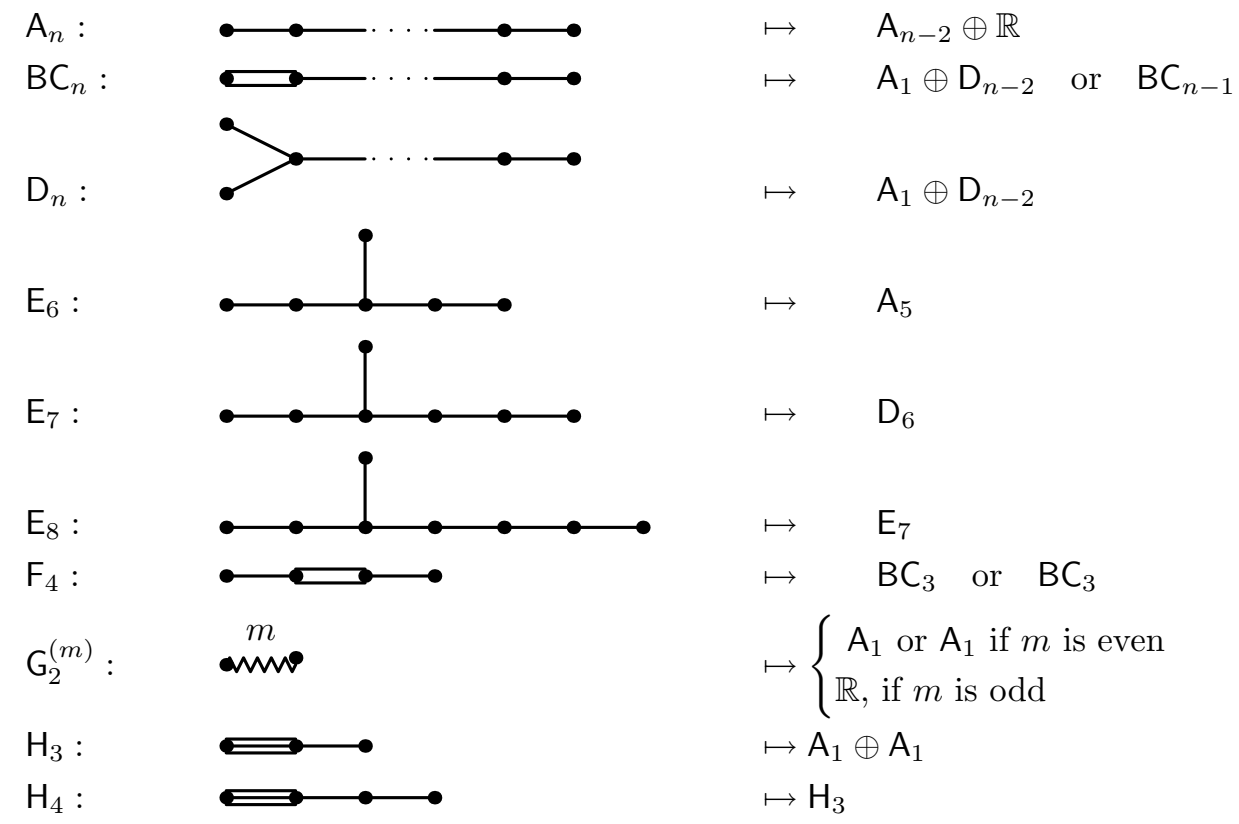

3.2. New equipment. All the strata of $B$ of dimension $<(n-2)$ are contained in the boundary of $B$. These strata of $B$ have their own equipments (in the sense of the Coxeter manifold $\mathbb{X}^{n-1}$ ).

Fix a boundary stratum $H$ of $B$, denote by $N_{H}$ the normal subspace to $H \subset \mathbb{X}^{n-1}$ at some point $y \in H$. The normal cone $D_{H} \subset N_{H}$ is the cone consisting of vectors looking inside $B$. Some of ( $n-2)$-dimensional strata (former hyperedges) $V_{\alpha}$ contain $H$ and thus we get the subdivision of the normal cone $D_{H}$ by tangent spaces to $(n-2)$-dimensional strata, see Fig 12

We wish to describe the equipment of $B \subset \mathbb{X}^{n-1}$ and the subdivisions of normal cones $D_{H}$.

3.3. Finite Coxeter groups. Let $\Gamma$ be a finite Coxeter group acting in $\mathbb{R}^{n}$. Let $\mathbb{X}_{j}^{n-1}$ be mirrors, let $v_{j}$ be the vectors orthogonal to the corresponding mirrors. For a vector $v_{k}$ denote by $R=R_{k}$ the set of all $i$ such that $v_{i}$ is orthogonal to $v_{k}$.

The reflection group $\Delta\left(\mathbb{X}_{k}^{n-1}\right)$ is generated by reflections with respect to mirrors $\mathbb{X}_{i}^{n-1}$, where $i$ ranges in $R$.

A. Let the Coxeter group $\Gamma$ be irreducible. We come to the list given in Table. Some comments:

1) $\mathrm{G}_{2}^{(m)}$ denotes the group of symmetries of a regular plane $m$-gon, $\mathbb{R}$ denotes the one-element group acting in $\mathbb{R}^{1}$; all other notations are standard, see [].

2) In some cases, there are two $\Gamma$-nonequivalent mirrors, then we write both possible variants.

The Rolling scheme (see 2.5) is the Coxeter scheme without even edges. 


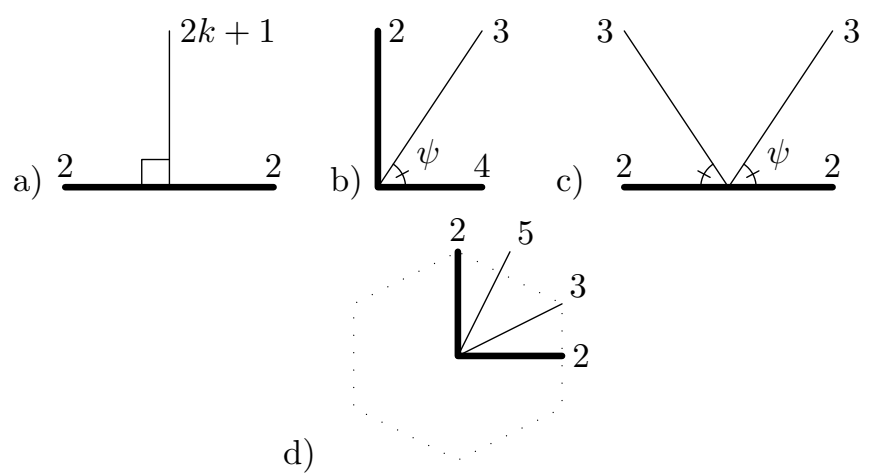

Figure 13. Subdivisions of a Coxeter polygon on the Lobachevsky plane (we also draw the labels on lines). There only 4 possible variants of meetings between lines of a subdivision and the boundary. Here $\tan \psi=\sqrt{2}$; for case d) see Fig. 5

In cases c) and d), the corresponding trihedral angle of the Andreev polytop is covered by our bended polygon.

ExAmPLE. a) For the Weyl chamber $E_{8}$, its complete development is the Weyl chamber $\mathrm{E}_{7}$.

b) For the Weyl chamber $\mathrm{BC}_{n}$, one of the facets is the Weyl chamber $\mathrm{BC}_{n-1}$. All the remaining facets are connected by the Rolling graph; the development is the Weyl chamber $\mathrm{A}_{1} \oplus \mathrm{D}_{n-2}$.

Proof of TABLE is a case-by-case examination of root systems, for groups $\mathrm{H}_{3}$, $\mathrm{H}_{4}$ proofs are given in figures above (on the other hand the reader can find a nice coordinate description of hypericosahedron in [8].

B. Let the Coxeter group $\Gamma$ be reducible,

$$
\Gamma=\Gamma_{1} \times \Gamma_{2} \times \ldots
$$

then its Weyl chamber is the product of Weyl chambers for the corresponding chambers $C=C_{1} \times C_{2} \times \ldots$ The Coxeter scheme of $\Gamma$ is the union of Coxeter scheme of $\Gamma_{j}$, hence the Rolling graph of $\Gamma$ is the union of Rolling graphs for $\Gamma_{j}$. Now we reduce one of factors $C_{j} \mapsto B_{j}$ according to the rules given in Table and get a Weyl chamber $B_{j} \times \prod_{i \neq j} C_{i}$.

3.4. Reduction of equipment. Let $H$ be an $(n-k)$-dimensional stratum of $C$ $(k \geqslant 3)$, let $\Gamma_{H}(C)$ be the corresponding Coxeter group, $\mathfrak{N}_{H}(C)$ be its chamber in the normal cone. Denote by $\Gamma_{H}(B)$ the corresponding group of the equipment of $B$ and by $\mathfrak{N}_{H}(B)$ the corresponding chamber in the normal cone.

Theorem 3.2. The group $\Gamma_{H}(B)$ is obtained by the reduction of the group $\Gamma_{H}(B)$ and the subdivision of $\mathfrak{N}_{H}(B)$ is a partial development of the Weyl chamber $\mathfrak{N}_{H}(C)$

Proof is obvious. We consider rollings of $C$ with fixed hyperdge $H$. 

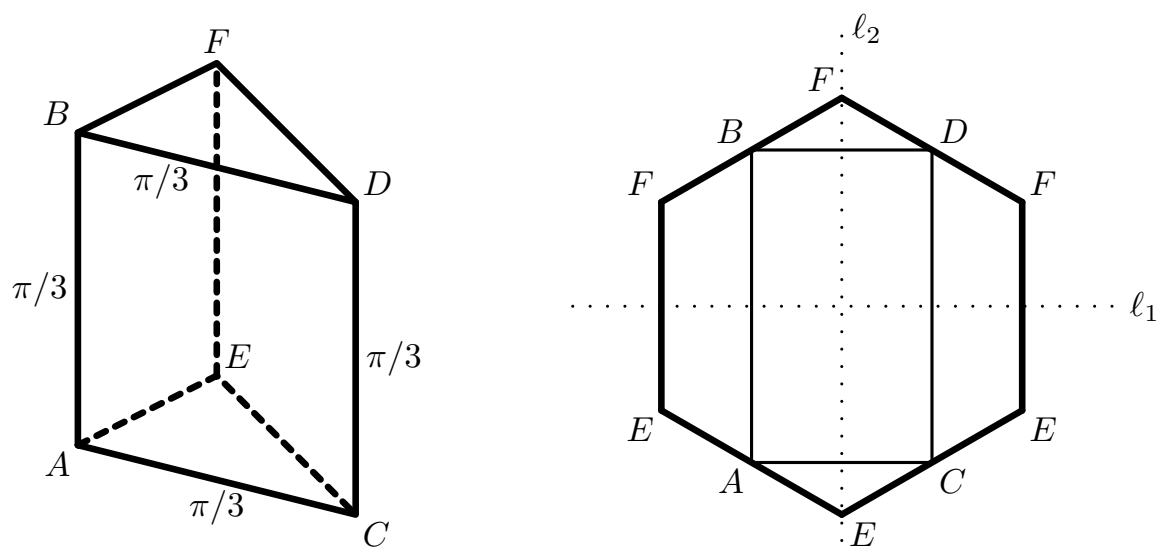

FiguRE 14. An example of Andreev polyhedron in $\mathbb{L}^{3}$; we label dihedral angles $\pi / 3$, all other dihedral angles are $\pi / 2$.

Its development is a (nonregular) 6 -gone, whose angles are $\pi / 2$. The lines $\ell_{1}, \ell_{2}$ are axes of symmetry. The polygonal curve $A B D C A$ is a billiard trajectory.

It is easy to reconstruct the lengths of edges of the prism from the combinatorial structure of the development and the billiard trajectory. Indeed, we know angles of the triangle $A E C$ and of the "trapezoids" YuN: trapezium? $A B F E$, and the equiangular quadrangle $A B D C$.

\section{Addendum. On elementary geometry of Andreev polyhedra}

4.1. Rolling of Andreev polyhedra and billiard trajectories in Coxeter polygons. First, our construction gives some information about developments of Andreev polyhedra.

Let us roll an Andreev polyhedron $\subset \mathbb{L}^{3}$ along a mirror $\simeq \mathbb{L}^{2}$. In this case, the chamber $B$ of a mirror is a convex plane Coxeter domain. By construction, $B$ is subdivided into several convex polygons by a certain family of lines.

Proposition 4.1. All the possible variants of meetings of lines of the subdivision and the boundary of $B$ are presented on Fig. 13.

Proof. We watch all the possible variants of reduction of 3-dimensional finite Coxeter groups to a mirror, figures a), b), c), d) correspond to $G_{2}^{2 k+1}, B_{3}, A_{3}=D_{3}$, $\mathrm{H}_{3}$ respectively.

Observation 4.2. The surface of an Andreev polyhedron is a glued from several bended Coxeter polygons; the rules of bending and the rules of glueing are very rigid.

EXAMPLES of rolling of a 3-dimensional Coxeter polyhedron are given on Figures 914

4.2. Example: Rolling along Andreev polyhedra. Secondly, take a Coxeter polyhedron in $\mathbb{L}^{4}$. Rolling it along 3-dimensional Lobachevsky space, we obtain a Coxeter polyhedron in $\mathbb{L}^{3}$ and also some strange subdivision of this polyhedron. 

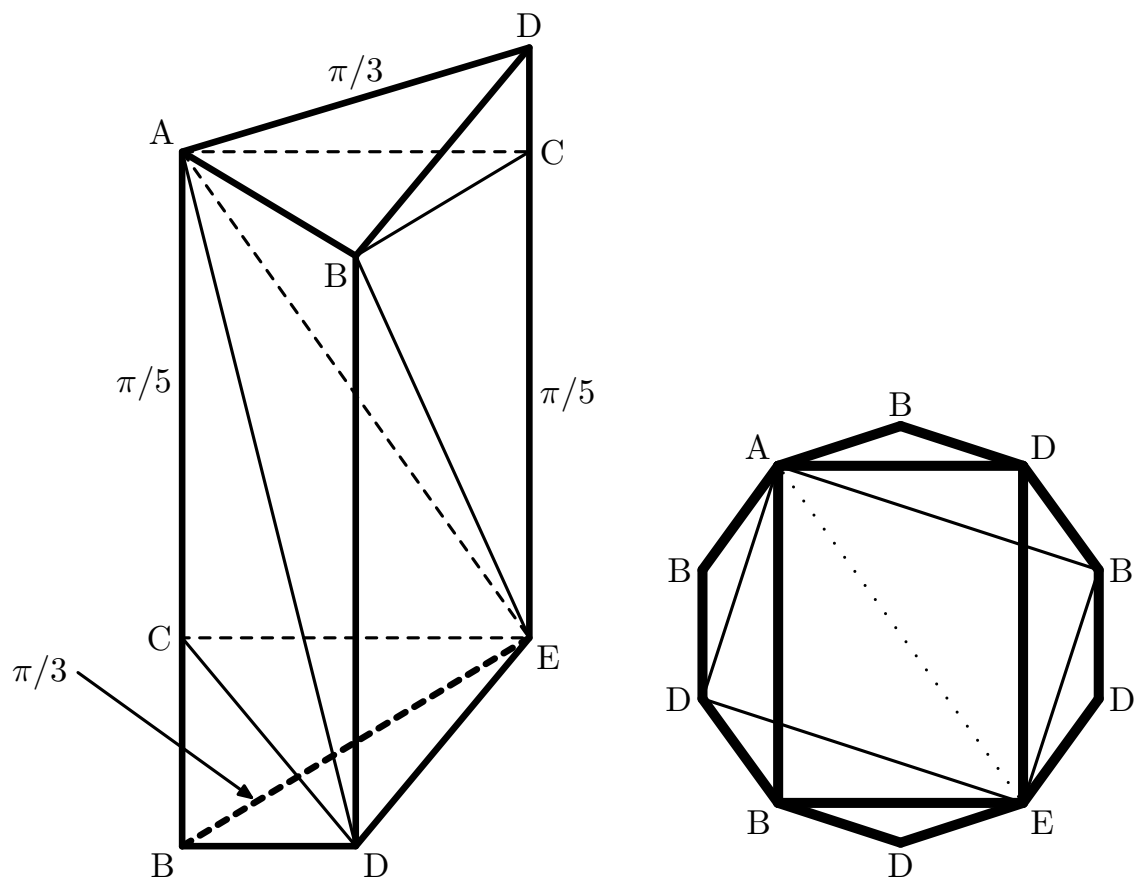

Figure 15. This prism in $\mathbb{L}^{3}$ is a complete development of the Coxeter simplex $A B C D E$ in $\mathbb{L}^{4}$ described in 4.2 It carries 2dimensional hyperedges of the initial simplex.

The development of the prism is the regular 10-gon with right angles (it also carries 1-dimensional strata of the 4-dimensional simplex).

We present an example.

Consider the simplex $\Sigma$ in $\mathbb{L}^{4}$ defined by the Coxeter scheme

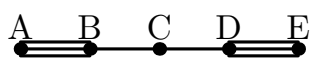

By $A, \ldots, E$ we denote the vertices of the simplex opposite to the corresponding faces.

Comments to Figure 15. The development of $\Sigma$ is a prism drawn in Fig 15. We write labels on the dihedral angles $\neq \pi / 2$. Below a "stratum" means a stratum of the tiling; in particular, the vertical "edge" $A B$ consists of two 1-dimensional strata $B C$ and $C A$ and three 0-dimensional, $A, B, C$.

1) It is a development. Hence any two strata (segments, triangles) having the same notation are equal (for instance $C D=C D, C E=C E, \triangle C B E=\triangle C B E$, etc.).

2) Each stratum (a vertex, a segment) is equipped with a Coxeter group (this group is visible from dihedral angles)

3) Subdivision of the normal cone $D_{H}$ to a stratum $H$ (a vertex, a segment) is determined by the reduction procedure from Subsection 3.3 
For instance, in the vertex $A$ we have the subdivision of the spherical triangle $\mathrm{H}_{3}$ drawn in Fig

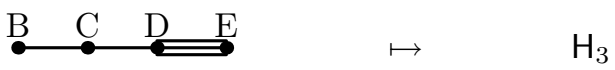

In the normal cone to the edge-stratum $D E$ of the prism, we have the icosahedral subdivision, see Fig. 8

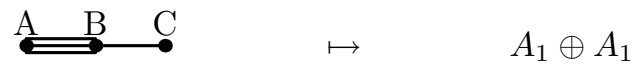

The normal cone to the segment $A E$ is drawn in Fig 4 in particular, both the angles of incidence $\operatorname{are} \arctan \sqrt{2}$,

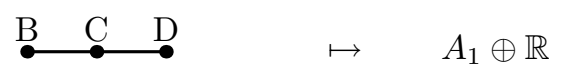

The "front" face $A B D B$ is orthogonal to the sections $C D E$ and $A D E$ (since the lines $C D$ and $A D$ of intersection are equipped with the group $\mathrm{A}_{1} \oplus \mathrm{A}_{1}$ ).

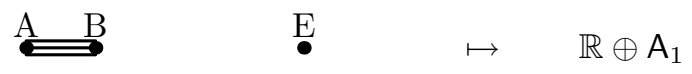

Etc., etc.

4) The prism has two planes of symmetry. This is by chance, partially this is induced by a symmetry of the initial Coxeter scheme 3 The latter symmetry implies the equality of strata:

$$
A B=D E, \quad A C=C E, \quad A D=B C, \quad B C=C D
$$

5) Our prism generates a reflection group in $\mathbb{L}^{3}$. The reader can easily imagine a neighborhood of our prism in $\mathbb{L}^{3}$. For instance, near the vertex $A$ we have the picture drawn in Fig 8

6) The development of the prism is a regular 10-gon having right angles; reflections of the "billiard trajectory" $A B E D A$ is of type d) on Fig. 13. The regularity property follows from reduction from $\mathbb{L}^{4}$, but it is not self-obvious from the picture of 3-dimensional prism. Obviously, diagonal $A B$ are orthogonal to diagonals $D E$ at points of intersection (see to the left side of the figure; but this is not a self-obvious property of this regular 10-gon).

7) We observe the second copy of the polygonal line $A D E B A$ in the development. Bending the 10-gon by YuN: by? this line, we obtain a prism congruent to our prism.

In fact, our 10-gon is the picture on the intersection of two mirrors, denote them by $Y_{1}, Y_{2}$. We can roll the simplex $\Sigma$ along each mirror $Y_{1}, Y_{2}$ and then we roll it again over the intersection $Y_{1} \cap Y_{2}$. We obtain two different pictures on the 10-gon and both are present on the Fig 15

\section{REFERENCES}

1. Aleksandrov A.D. Convex polyhedra. Gosudarstv. Izdat. Tehn.-Teor. Lit., Moscow, 1950; German translation: Akademie-Verlag, Berlin, 1958; English translation: Springer, 2005

2. Alekseevky, D.V., Kriegl A., Losik M., Michor, P.W., The Riemannian geometry of orbit spaces. The metric, geodesics, and integrable systems, Publ. Math. 62 (2003), no. 3-4, arXiv:math.DG/0102159.

3. Alexeevky D.V., Kriegl, A., Losik, M., Michor P.W., Reflection groups on Riemannian manifolds, Annali Mat. pur. appl. 186 (2007), no. 1, 25-58.

4. Alekseevskij, D. V.; Vinberg, E. B.; Solodovnikov, A. S. Geometry of spaces of constant curvature. Geometry, II, 1-138, Encyclopaedia Math. Sci., 29, Springer, Berlin, 1993.

${ }^{5}$ There are two diagonals $A B$. 
5. Andreev, E. M. Convex polyhedra in Lobachevsky spaces. Mat. Sb. (N.S.) 81 (123) $1970445-$ 478. English transl.: Math. USSR Sb., 10(5), 1970, 413-440

6. Andreev, E. M. Convex polyhedra of finite volume in Lobachevsky space. Mat. Sb. (N.S.) 83 (125) 1970 256-260. English transl. in Math. USSR Sb., 12(3), 225-259 (1971)

7. Andreev, E. M. On intersections of planes bounded an acute angle polyhedron., Math. Notes, 8 (1971) 761-764.

8. Bourbaki, N. Elements de mathematique. Fasc. XXXIV. Groupes et algebres de Lie. Chapitre IV: Groupes de Coxeter et systhemes de Tits. Chapitre V: Groupes engendres par des reflexions. Chapitre VI: systemes de racines. Hermann, Paris 1968.

9. Davis, M. W. Coxeter groups and aspherical manifolds. Algebraic topology, Aarhus 1982 (Aarhus, 1982), 197-221, Lecture Notes in Math., 1051, Springer, Berlin, 1984

10. Davis, M. Groups generated by reflections and aspherical manifolds not covered by Euclidean space. Ann. Math., 117 (1983), 293-324

11. Davis, M. Geometry and topology of Coxeter manifolds. Princeton Univ. Press, 2007.

12. Felikson A., Tumarkin P. On compact hyperbolic Coxeter d-polytops with $(d+4)$ facets. Preprint, available via http://xxx.arHiv.org//math.MG/0510238

13. Khovanskii, A. Combinatorics of sections of polytopes and Coxeter groups in Lobachevsky spaces. The Coxeter legacy, 129-157, Amer. Math. Soc., Providence, RI, 2006.

14. Hodgson, C. D. Deduction of Andreev's theorem from Rivin's characterization of convex hyperbolic polyhedra. Topology '90 (Columbus, OH, 1990), 185-193, Ohio State Univ. Math. Res. Inst. Publ., 1, de Gruyter, Berlin, 1992.

15. Lyusternik, L. A. Convex figures and polyhedra. Translated and adapted from the first Russian edition (1956) by Donald L. Barnett D. C. Heath and Co., Boston, Mass. 1966

16. Rivin, I., Hodgson, C. D.; A characterization of compact convex polyhedra in hyperbolic 3space. Invent. Math. 111 (1993), no. 1, 77-111.

17. Rivin, I. A characterization of ideal polyhedra in hyperbolic 3-space. Ann. of Math. (2) 143 (1996), no. 1, 51-70.

18. Tits, J. Groupes et geometrie de Coxeter, I.H.E.S., 1961, mimeographed notes.

19. Thurston, W., The Geometry and Topology of Three-Manifolds. The text is available via http://www.msri.org/communications/books/gt3m

20. Vinberg, E. B. Discrete linear groups that are generated by reflections. Math. USSR Izvestia 5 (1971), 1083-1119.

21. Vinberg, E.B. Hyperbolic reflection groups, Russian Math. Surveys 40 (1985), 31-75.

22. Vinberg, E. B.; Shvartsman, O. V. Discrete groups of motions of spaces of constant curvature. Geometry, II, 139-248, Encyclopaedia Math. Sci., 29, Springer, Berlin, 1993.

D. V. Alekseevskij: School of Mathematics, Edinburgh University, Edinburgh, EH9 3JZ, UNITED KingDOM

E-mail address: d.aleksee@ed.ac.uk

P. W. Michor: Fakultät für Mathematik, Universität Wien, Nordbergstrasse 15, A1090 Wien, Austria; and: Erwin Schrödinger Institute of Mathematical Physics, BoltzMANngasse 9, A-1090 Wien, Austria. YUN: : IS IT CORRECT??

E-mail address: Peter.Michor@esi.ac.at

Yu. A. Neretin: Fakultät für Mathematik, Universität Wien, Nordbergstrasse 15, A-1090 Wien, Austria and Group of Math.Physics, ITEP, B.Cheremushkinskaya, 25, Moscow, 117259, Russia , and MechMath Department, Moscow State University, Vorob'yovy Gory, Moscow, Russia

E-mail address: neretin@mccme.ru 